\title{
TU/e EmonONEN

\section{Two parallel insurance lines with simultaneous arrivals and risks correlated with inter-arrival times}

\section{Citation for published version (APA):}

Badila, E. S., Boxma, O. J., \& Resing, J. A. C. (2015). Two parallel insurance lines with simultaneous arrivals and risks correlated with inter-arrival times. Insurance: Mathematics and Economics, 61, 48-61.

https://doi.org/10.1016/j.insmatheco.2014.12.003

DOI:

10.1016/j.insmatheco.2014.12.003

Document status and date:

Published: 01/01/2015

\section{Document Version:}

Publisher's PDF, also known as Version of Record (includes final page, issue and volume numbers)

\section{Please check the document version of this publication:}

- A submitted manuscript is the version of the article upon submission and before peer-review. There can be important differences between the submitted version and the official published version of record. People interested in the research are advised to contact the author for the final version of the publication, or visit the $\mathrm{DOI}$ to the publisher's website.

- The final author version and the galley proof are versions of the publication after peer review.

- The final published version features the final layout of the paper including the volume, issue and page numbers.

Link to publication

\section{General rights}

Copyright and moral rights for the publications made accessible in the public portal are retained by the authors and/or other copyright owners and it is a condition of accessing publications that users recognise and abide by the legal requirements associated with these rights.

- Users may download and print one copy of any publication from the public portal for the purpose of private study or research.

- You may not further distribute the material or use it for any profit-making activity or commercial gain

- You may freely distribute the URL identifying the publication in the public portal.

If the publication is distributed under the terms of Article 25fa of the Dutch Copyright Act, indicated by the "Taverne" license above, please follow below link for the End User Agreement:

www.tue.nl/taverne

Take down policy

If you believe that this document breaches copyright please contact us at:

openaccess@tue.nl

providing details and we will investigate your claim. 


\title{
Two parallel insurance lines with simultaneous arrivals and risks correlated with inter-arrival times
}

\author{
E.S. Badila*, O.J. Boxma, J.A.C. Resing \\ Department of Mathematics and Computer Science, Eindhoven University of Technology, P.O. Box 513, 5600 MB Eindhoven, The Netherlands
}

\section{A R T I C L E I N F O}

Article history:

Received May 2014

Received in revised form

November 2014

Accepted 7 December 2014

Available online 15 December 2014

\section{MSC:}

primary $91 \mathrm{~B} 30$

$60 \mathrm{~K} 25$

\section{Keywords:}

Insurance risk

Multivariate ruin probability

Reinsurance

Dependence

Duality

Parallel queues

Bivariate waiting time

\begin{abstract}
A B S T R A C T
We investigate an insurance risk model that consists of two reserves which receive income at fixed rates. Claims are being requested at random epochs from each reserve and the interclaim times are generally distributed. The two reserves are coupled in the sense that at a claim arrival epoch, claims are being requested from both reserves and the amounts requested are correlated. In addition, the claim amounts are correlated with the time elapsed since the previous claim arrival.

We focus on the probability that this bivariate reserve process survives indefinitely. The infinitehorizon survival problem is shown to be related to the problem of determining the equilibrium distribution of a random walk with vector-valued increments with 'reflecting' boundary. This reflected random walk is actually the waiting time process in a queueing system dual to the bivariate ruin process.

Under assumptions on the arrival process and the claim amounts, and using Wiener-Hopf factorization with one parameter, we explicitly determine the Laplace-Stieltjes transform of the survival function, c.q., the two-dimensional equilibrium waiting time distribution.

Finally, the bivariate transforms are evaluated for some examples, including for proportional reinsurance, and the bivariate ruin functions are numerically calculated using an efficient inversion scheme.
\end{abstract}

(c) 2014 Elsevier B.V. All rights reserved.

\section{Introduction}

We study a two-dimensional ruin problem for a bivariate risk reserve process in which claims are simultaneously requested from both reserves. The amounts of two simultaneously arriving claims may be correlated, and may also be correlated with the time elapsed since the previous claim arrival. Under assumptions on the arrival process and the claim sizes, we explicitly determine the Laplace-Stieltjes transform (LST) of the survival function.

Studies of multidimensional risk reserve processes are scarce in the insurance literature, although results about risk measures related to such models are highly relevant both from a theoretical and a practitioner's perspective. Multivariate ruin problems are relevant because they give insight into the behaviour of risk measures under various types of correlations between the insurance lines. One example is presented by multiple insurance lines within the same company which are interacting with each other as they evolve in time, via, say, coupled income rates. Another typical example is an umbrella type of insurance model, where a claim occurrence event generates multiple types of claims which may be

\footnotetext{
* Corresponding author.

E-mail addresses: e.s.badila@tue.nl (E.S. Badila), o.j.boxma@tue.nl (O.J. Boxma), resing@win.tue.nl (J.A.C. Resing).
}

correlated, and each type $i$ claim is paid from its corresponding reserve $R^{(i)}$, such as car insurance together with health insurance or insurance against earthquakes. Yet another class of models is related to reinsurance problems, where a claim is shared between the insurer and one or more reinsurers.

In the existing risk and insurance literature, there are not many approaches towards analysing such complicated multidimensional models. A first attempt to assess multivariate risk measures can be found in the paper of Sundt (1999) about developing multivariate Panjer recursions which are then used to compute the distribution of the aggregate claim process, assuming simultaneous claim events and discrete claim sizes. Other approaches are deriving integro-differential equations for the various measures of risk and then iterating these equations to find numerical approximations (Chan et al., 2003; Gong et al., 2012), or computing bounds for the different types of ruin probabilities that can occur in a setting where more than one insurance line is considered (see Cai and $\mathrm{Li}$, 2005 which considers multivariate phase-type claims). It is worth mentioning that very few papers (like Avram et al., 2008a; Badescu et al., 2011; Badila et al., 2014), analytically determine, e.g., the ruin probability for insurance models with more than one reserve.

In an attempt to solve the integro-differential equations that arise from such models, Chan et al. (2003) derive a so called 'boundary value problem' of a Riemann-Hilbert type for the bivariate 
Laplace transform of the joint survival function (see Badila et al., 2014 for details about such problems arising in the context of risk and queueing theory and the book (Cohen and Boxma, 1983) for an extended analysis of similar models in queueing). However, Chan et al. (2003) do not solve this functional equation for the Laplace transform. The law of the bivariate reserve process usually considered in the above mentioned works is that of a compound Poisson process with vector-valued jumps supported on the negative quadrant in $\mathbb{R}^{2}$, conditioned to start at some positive reserve, and linearly drifting along a direction vector that belongs to the positive quadrant. In Badila et al. (2014) a similar functional equation is taken as a departure point, and it is explained how one can find transforms of ruin related performance measures via solutions of the above mentioned boundary value problems. It is also shown that the boundary value problem has an explicit solution in terms of transforms, if the claim sizes are ordered. A special, important case is the setting of proportional reinsurance, which was studied in Avram et al. (2008a,b). There it is assumed that there is a single arrival process, and the claims are proportionally split among two reserves. In this case, the two-dimensional exit (ruin) problem becomes a one-dimensional first-passage problem above a piecewise linear barrier. Badescu et al. (2011) have extended this model by allowing a dedicated arrival stream of claims into only one of the insurance lines. They show that the transform of the time to ruin of at least one of the reserve processes can be derived using similar ideas as in Avram et al. (2008a).

The approach we take in this paper generalizes ideas in Badila et al. $(2013,2014)$, and will allow us to extend those two studies. In Section 3 we derive a similar functional equation as in Badila et al. (2014) for the survival function related to a 2-dimensional reserve process, but unlike Badila et al. (2014) we do not assume that the claim intervals are exponentially distributed. Furthermore we assume the claim size vector to be correlated with the time elapsed since the previous arrival. Such a correlation is quite natural; e.g., a claim event that generates very large claims could be subjected to additional administrative/regulatory delays. The type of correlation between the inter-arrival time and the vector of claim sizes is an extension to two dimensions of the dependence structure studied in Badila et al. (2013) for a generalized SparreAndersen model. It involves making a rationality assumption regarding the trivariate LST of inter-arrival time and claim size vector (Assumption 2.1), which extends the case where the vector with the aforementioned components has a multivariate phase type distribution (MPH). In addition, we also make the assumption that the claim sizes are a.s. ordered (Assumption 2.2). Under these assumptions, we obtain our main result: An explicit expression for the (LST of the) two-dimensional survival function, for a large class of vectors of interclaim times and claim amounts of both reserves.

The paper is organized in the following way. In Section 2 we describe the model and present the main assumptions we will be working with. Section 3 is dedicated to some general theory for random walks in the plane; we show a useful relation between the two-dimensional risk reserve process and a version of the random walk which has the boundary of the nonnegative quadrant in $\mathbb{R}^{2}$ as an impenetrable barrier. This relation also makes it clear that determining the survival function is equivalent with determining the two-dimensional waiting time distribution in a dual two-queue two-server queueing model with simultaneous arrivals of customers at both queues. With the help of the random walk/queueing process we derive, in Section 4, a stochastic recursion for the LST of the finite horizon survival function. In Section 5 we resolve the stationary version of this stochastic recursion, Formula (5.1). The key tool used is a one-parameter Wiener-Hopf factorization of the bivariate kernel appearing in Eq. (5.1). More precisely, the Wiener-Hopf factors will depend on one parameter, which is the first argument of that bivariate kernel; see Proposition 2 in Section 5. The main result, Theorem 2, gives the LST of the survival function, or equivalently the stationary distribution of the waiting time/reflected random walk inside the positive quadrant (see also Remark 3).

In Section 6 we explain how to calculate the transform obtained in Theorem 2 for some examples, and we numerically calculate the ruin functions/waiting time distributions using an efficient inversion algorithm of den Iseger (2006). Finally we also point out that the numerical results suggest that the ruin functions appear to be stochastically ordered for various types of correlations between inter-arrival times and claim sizes, a positive correlation leading to smaller ruin probabilities.

\section{Model description}

Let us begin with the general assumptions on the two reserves. The reserves start with non-negative initial capital $\left(u^{(1)}, u^{(2)}\right)$; as long as there are no arrivals, the reserves increase linearly with positive rates $\left(c^{(1)}, c^{(2)}\right)$. At the $n$th claim arrival epoch, claim sizes $B_{n}^{(1)}$ and $B_{n}^{(2)}$ are respectively requested from each reserve. The time between the $(n-1)$ th and $n$th claim arrival is denoted by $A_{n}$. The sequence $\left\{A_{n}, B_{n}^{(1)}, B_{n}^{(2)}\right\}_{n \geq 1}$, is assumed to be an i.i.d. sequence, but within a triple, $\left(A_{n}, B_{n}^{(1)}, B_{n}^{(2)}\right)$ are allowed to be correlated. We will use $A, B^{(1)}, B^{(2)}$ respectively for the generic inter-arrival time and claim sizes. In the above-described very general set-up, the following assumption will allow us to explicitly determine the ruin/survival probabilities by using Wiener-Hopf factorization:

Assumption 2.1 (On the Joint Transform of $\mathbf{A}, \mathbf{B}^{(1)}, \mathbf{B}^{(2)}$ ). The triple transform

$H\left(q_{0}, q_{1}, q_{2}\right):=\mathbb{E} e^{-q_{0} A-q_{1} B^{(1)}-q_{2} B^{(2)}}$

is a rational function in $q_{i}, i=0,1,2$, i.e., it has representation $\frac{N\left(q_{0}, q_{1}, q_{2}\right)}{D\left(q_{0}, q_{1}, q_{2}\right)}$ such that $N\left(q_{0}, q_{1}, q_{2}\right)$ and $D\left(q_{0}, q_{1}, q_{2}\right)$ are polynomials in the variables $q_{i}, i=0,1,2$.

$N\left(q_{0}, q_{1}, q_{2}\right)$ and $D\left(q_{0}, q_{1}, q_{2}\right)$ must satisfy some conditions, because their ratio is a transform, such as,

$$
\lim _{\left|q_{0}\right| \rightarrow \infty, \mathcal{R} e q_{0}>0} H\left(q_{0}, q_{1}, q_{2}\right)=\mathbb{E}\left[e^{-q_{1} B^{(1)}-q_{2} B^{(2)}} 1_{\{A=0\}}\right],
$$

with $1_{\{E\}}$ the indicator function of event $E$. We can assume without loss of generality that $A>0$ a.s. Because of the above limit, this means the degree of $N$ as a polynomial in $q_{0}, N_{q_{1}, q_{2}}\left(q_{0}\right)$, is strictly less than the degree of $D$ as a polynomial in $q_{0}: D_{q_{1}, q_{2}}\left(q_{0}\right)$, for all $q_{1}$ and $q_{2}$.

Remark 1. The class of rational multivariate Laplace-Stieltjes transforms contains the class of LSTs of multivariate Phase-Type distributions (MPH); see Bladt and Nielsen (2010), where the rational transform class is called multivariate matrix-exponential (MME). All of the well-known classes of multivariate Phase-Type distributions are MME. Actually, all the examples we will present are MPH distributions with a specific correlation structure which are a special case of Kulkarni's MPH* class (Kulkarni, 1989). There is no point in restricting ourselves to any of these subclasses. We will fully exploit the algebraic representation of rational functions in order to derive explicitly the transforms of the two-dimensional survival/ruin functions.

The reserve process $\bar{R}_{t}=\left(\bar{R}_{t}^{(1)}, \bar{R}_{t}^{(2)}\right)$ evolves as

$\bar{R}_{t}=\bar{u}+c t-\sum_{i=1}^{n(t)} B_{i}, \quad$ where $\bar{u}:=\left(\bar{u}^{(1)}, \bar{u}^{(2)}\right), c:=\left(c^{(1)}, c^{(2)}\right)$,

$B_{i}:=\left(B_{i}^{(1)}, B_{i}^{(2)}\right)$, 
and $n(t)$ is the number of arrivals before $t$. We use the notation $\bar{R}$ with a bar because later on we want to scale the reserve process by dividing $\bar{R}_{t}^{(i)}$ by $c^{(i)}$, using the notation $R_{t}^{(i)}$ without a bar for the resulting process. In this model, the two reserves are correlated due to simultaneous claim arrivals and due to correlations that may exist in the claim size vector $\left(B^{(1)}, B^{(2)}\right)$.

This paper is concerned with measuring the event that both reserve processes survive indefinitely, i.e., we aim to determine the survival function

$$
\begin{aligned}
& \bar{F}^{s}\left(\bar{u}^{(1)}, \bar{u}^{(2)}\right) \\
& \quad:=\mathbb{P}\left(\bar{R}_{t}^{(1)} \geq 0, \forall t>0 \text { and } \bar{R}_{t}^{(2)} \geq 0, \forall t>0 \mid \bar{R}_{0}=\left(\bar{u}^{(1)}, \bar{u}^{(2)}\right)\right) .
\end{aligned}
$$

In terms of times to ruin, $\bar{F}^{s}$ is related to the first time at least one of the two insurance lines is ruined,

$$
\begin{aligned}
\bar{\tau}_{\wedge}\left(\bar{u}^{(1)}, \bar{u}^{(2)}\right) & =\inf \left\{t ; \min \left(\bar{R}_{t}^{(1)}, \bar{R}_{t}^{(2)}\right)<0\right\} \\
& =\bar{\tau}^{(1)}\left(\bar{u}^{(1)}\right) \wedge \bar{\tau}^{(2)}\left(\bar{u}^{(2)}\right),
\end{aligned}
$$

where $\bar{\tau}^{(i)}\left(u^{(i)}\right)$ are the marginal times to ruin, $i=1,2$. In particular,

$\bar{F}^{s}\left(\bar{u}^{(1)}, \bar{u}^{(2)}\right)=1-\mathbb{P}\left(\bar{\tau}_{\wedge}\left(\bar{u}^{(1)}, \bar{u}^{(2)}\right)<\infty\right)$.

We can also define the first time at which both insurance lines are ruined:

$\bar{\tau}_{\vee}\left(\bar{u}^{(1)}, \bar{u}^{(2)}\right)=\bar{\tau}^{(1)}\left(\bar{u}^{(1)}\right) \vee \bar{\tau}^{(2)}\left(\bar{u}^{(2)}\right)$.

It is similarly related to the probability that at least one of the two reserves survives indefinitely,

$$
\begin{aligned}
& \bar{F}_{O R}^{s}\left(\bar{u}^{(1)}, \bar{u}^{(2)}\right) \\
& \quad:=\mathbb{P}\left(\bar{R}_{t}^{(1)} \geq 0, \forall t>0 \text { or } \bar{R}_{t}^{(2)} \geq 0, \forall t>0 \mid \bar{R}_{0}=\left(\bar{u}^{(1)}, \bar{u}^{(2)}\right)\right),
\end{aligned}
$$

by

$\bar{F}_{O R}^{s}\left(\bar{u}^{(1)}, \bar{u}^{(2)}\right)=1-\mathbb{P}\left(\bar{\tau}_{\vee}\left(\bar{u}^{(1)}, \bar{u}^{(2)}\right)<\infty\right)$.

Notice that $\bar{\tau}_{\vee}$ is not the same as $\inf \left\{t ; \max \left(\bar{R}_{t}^{(1)}, \bar{R}_{t}^{(2)}\right)<0\right\}$, that is, joint ruin may not happen simultaneously.

The above survival functions are then related by

$\bar{F}_{O R}^{s}\left(\bar{u}^{(1)}, \bar{u}^{(2)}\right)=\bar{F}^{s}\left(\bar{u}^{(1)}, \infty\right)+\bar{F}^{s}\left(\infty, \bar{u}^{(2)}\right)-\bar{F}^{s}\left(\bar{u}^{(1)}, \bar{u}^{(2)}\right)$,

where $\bar{F}^{s}\left(\bar{u}^{(1)}, \infty\right)$ and $\bar{F}^{s}\left(\infty, \bar{u}^{(2)}\right)$ are the marginal survival functions. Moreover, we also have $\bar{F}^{i, j}\left(\bar{u}^{(1)}, \bar{u}^{(2)}\right)$, the probability that reserve $i$ survives indefinitely, while reserve $j$ ruins, for $i, j=$ $1,2, i \neq j$.

$\bar{F}^{1,2}\left(\bar{u}^{(1)}, \bar{u}^{(2)}\right)=\bar{F}^{s}\left(\bar{u}^{(1)}, \infty\right)-\bar{F}^{s}\left(\bar{u}^{(1)}, \bar{u}^{(2)}\right)$,

and similarly for $\bar{F}^{2,1}\left(\bar{u}^{(1)}, \bar{u}^{(2)}\right)$. In view of the above, it suffices to determine $\bar{F}^{s}\left(\bar{u}^{(1)}, \bar{u}^{(2)}\right)$ in order to obtain all the other survival/ruin functions.

Remark 2. For the study of survival functions, we can normalize the reserve processes by their respective income rates. The survival function is preserved, with the starting capital scaled accordingly. To be more precise, let

$R_{t}^{(i)}=\bar{u}^{(i)} / c^{(i)}+t-\sum_{k=1}^{n(t)} B_{k}^{(i)} / c^{(i)}, \quad i=1,2$.

Since $R_{t}^{(i)} \geq 0 \Leftrightarrow \bar{R}_{t}^{(i)} \geq 0$, for $\bar{\tau}_{\wedge}, \tau_{\wedge}$ the exit times of $\bar{R}_{t}$ respectively $R_{t}$ from the non-negative quadrant, we have the relation

$\tau_{\wedge}\left(u^{(1)}, u^{(2)}\right)=\bar{\tau}_{\wedge}\left(c^{(1)} u^{(1)}, c^{(2)} u^{(2)}\right)$

and then also $F^{s}\left(u^{(1)}, u^{(2)}\right)=\bar{F}^{s}\left(c^{(1)} u^{(1)}, c^{(2)} u^{(2)}\right)$, with $F^{s}$ the survival function of the scaled process.

This means that for our purposes it suffices to study the process $R_{t}$ and the associated survival functions $F^{s}\left(u^{(1)}, u^{(2)}\right) . F_{O R}^{s}$ and the complementary ruin time $\tau_{\vee}$ are similarly defined.
The main idea of our approach is to exploit the fact that the embedded process at arrival epochs of claims is a random walk in the plane with increments $\left(A_{n}-B_{n}^{(1)} / c^{(1)}, A_{n}-B_{n}^{(2)} / c^{(2)}\right)$, conditioned on starting at $\left(u^{(1)}, u^{(2)}\right)$. The different ways in which the reserve process can be ruined correspond to the possible positions of the random walk

$R_{t_{n}}:=u+\sum_{i=1}^{n} X_{i}$

$X_{n}:=\left(X_{n}^{(1)}, X_{n}^{(2)}\right):=\left(A_{n}-B_{n}^{(1)} / c^{(1)}, A_{n}-B_{n}^{(2)} / c^{(2)}\right)$,

at the time of exit from the non-negative quadrant. Here and below, $t_{n}$ denotes the $n$th arrival epoch.

This is a difficult model to analyse in full generality; in particular, it is much more general than the 2-dimensional ruin process described in Chan et al. (2003) and Badila et al. (2014). However, in the case that the claims in insurance line 1 are larger than those in insurance line 2, we are able to determine the two-dimensional survival function.

Assumption 2.2 (Ordering Assumption). For a generic claim event $\left(B^{(1)}, B^{(2)}\right)$ :

$B^{(1)} / c^{(1)} \geq B^{(2)} / c^{(2)} \quad$ a.s.

A special, important example for this ordering assumption is the case when there is a single arrival process such that the common claim is partitioned into fixed proportions $(\alpha, 1-\alpha)$, and we can always take w.l.o.g. $\alpha \in[1 / 2,1]$ ( $\alpha$ may even be a random variable with this interval as support). This special case then significantly generalizes the setting in Avram et al. (2008a), where it is assumed that the common arrival process is compound Poisson (and in particular the inter-arrival times are independent of the claim sizes).

One can go a step further and assume there is a dedicated renewal-arrival stream of claims into the line which pays the greater share, say $\alpha$. This is in line with the assumption in Badescu et al. (2011) of a dedicated Poissonian stream of claims, and extends it, once we combine it with Assumption 2.1. Clearly this is not a proportional reinsurance problem anymore. From a mathematical perspective, the analysis is more insightful than if one would just assume proportionality.

We take the following approach: using a duality argument (Section 3 ), we derive a recursive equation for the survival function (Section 4). Using complex function theory, and under Assumptions 2.1 and 2.2, we solve the functional equation that corresponds to the stochastic recursion in terms of survival function LSTs (Section 5).

\section{Duality}

It turns out that it is more fruitful to regard the survival function defined in the previous section as a distribution rather than as a function. In this section we point out that the finite horizon survival functions $F_{n}^{s}$ can be seen as the c.d.f.s of the so called reflected version of the random walk. This reflected version satisfies a recursive equation that is then exploited in the subsequent sections in order to calculate the infinite horizon survival function.

For a one-dimensional random walk it is well known that its running maximum has the same distribution as the reflected version of the random walk:

$$
\sup _{0 \leq i \leq n} S_{i}^{(1)} \stackrel{d}{=} S_{n}^{(1)}-\inf _{0 \leq i \leq n} S_{i}^{(1)}
$$

where $\stackrel{d}{=}$ denotes equality in distribution. Here we could, e.g., take $S_{n}^{(1)}=-\sum_{i=1}^{n} X_{i}^{(1)}$, where $X_{i}^{(1)}=A_{i}-B_{i}^{(1)} / c^{(1)}$ as defined in (2.3) 
for the one-dimensional first risk reserve process. In this section we show that this relation is still valid for the embedded random walk related to the general bivariate process $\left\{-R_{t}\right\}_{t \geq 0}$, without having enforced the assumptions from the previous section. This relation is further used to derive a recursion for the two-dimensional transform of the survival function. We also give an interpretation of this recursion in terms of excursions away from the running maximum of the reserve process $\left\{R_{t}\right\}_{t \geq 0}$.

Throughout this section we will work with the natural order on $\mathbb{R}^{2}$. For $x:=\left(x^{(1)}, x^{(2)}\right), y:=\left(y^{(1)}, y^{(2)}\right) \in \mathbb{R}^{2}$, denote

$x \preceq y \Leftrightarrow x^{(1)} \leq y^{(1)}, x^{(2)} \leq y^{(2)}$.

To keep notations short, set

$x \vee y:=\left(\max \left(x^{(1)}, y^{(1)}\right), \max \left(x^{(2)}, y^{(2)}\right)\right)$,

$x \wedge y:=\left(\min \left(x^{(1)}, y^{(1)}\right), \min \left(x^{(2)}, y^{(2)}\right)\right)$.

Denote by $S_{n}:=\sum_{i=1}^{n}\left(-X_{i}\right), n \geq 1$ and $S_{0}=0$, the origin of $\mathbb{R}^{2}$. $X_{n}$ was defined in (2.3). Ruin can only occur at arrival epochs, and since arrivals are simultaneous, we can introduce the maximum aggregate loss up to the $n$th arrival epoch $M_{n}:=\bigvee_{i=0}^{n} S_{i}$, so that we have the following relation for $\tau_{\wedge}$, the scaled version of the exit time that was defined in (2.2):

$\left\{\tau_{\wedge}\left(u^{(1)}, u^{(2)}\right)>t_{n}\right\}=\left\{M_{n} \preceq\left(u^{(1)}, u^{(2)}\right)\right\}$,

for $t_{n}$ the $n$th arrival epoch. Note also that $\tau_{\wedge}$ can now be rewritten in terms of the order relation ' $\succeq$ ':

$\tau_{\wedge}\left(u^{(1)}, u^{(2)}\right)=\inf \left\{t_{n} ; R_{t_{n}} \nsucceq 0 \mid R_{0}=\left(u^{(1)}, u^{(2)}\right)\right\}$.

The duality approach described in the following, allows one to obtain a recursion for the two-dimensional survival function by turning this 'exit problem' for the embedded random walk into a so-called 'reflection problem' (Theorem 1). This recursion is then solved in Section 5 under Assumptions 2.1 and 2.2. The behaviour of the bivariate reserve process $\left\{R_{t}\right\}_{t \geq 0}$ is similar to the behaviour of the reserve process studied in Avram et al. (2008a), one of the main differences being that in our set-up this is not a Markov process anymore.

For fixed $n$ let $S_{k}^{*}:=S_{n}-S_{n-k}, k=0, \ldots, n$, so that $S_{0}^{*}=$ $0, S_{1}^{*}=-X_{n}, S_{2}^{*}=-X_{n}-X_{n-1}$, etc. Thus $S_{i}^{*}, i \leq n$, is obtained from $S_{i}$ by circularly permuting its increments. The following lemma is the 2-dimensional version of (3.1). It remains valid in any number of dimensions by making some straightforward modifications.

Lemma 1. For all $n \geq 0$,

$M_{n} \stackrel{d}{=} S_{n}-\bigwedge_{i=0}^{n} S_{i}$

Proof. We can write

$S_{n}-\bigwedge_{i=0}^{n} S_{i}=S_{n}+\bigvee_{i=0}^{n}\left(-S_{i}\right)=\bigvee_{i=0}^{n}\left(S_{n}-S_{i}\right)=\bigvee_{i=0}^{n} S_{n-i}^{*}$.

Here we used the relation: $-(x \wedge y)=(-x) \vee(-y), x, y \in \mathbb{R}^{2}$. The above are all sample-path identities. The final step is to remark that the joint distribution of $\left(S_{1}^{*}, \ldots, S_{n}^{*}\right)$ is the same as the distribution of $\left(S_{1}, \ldots, S_{n}\right)$ because the increments $X_{i}$ are i.i.d., and the proof is complete.

Denote $W_{n}:=S_{n}-\bigwedge_{i=0}^{n} S_{i}, n \geq 0$, the random walk reflected inside the nonnegative quadrant. An important step in our analysis of the survival function is the following recursion for the sequence $\left(W_{n}\right)_{n \geq 0}$.
Proposition 1. The sequence $\left(W_{n}\right)_{n \geq 0}$ satisfies the following recursion path-wise:

$W_{n+1}=\left(W_{n}-X_{n+1}\right) \vee 0$,

and initial condition $W_{0}=0$.

Roughly speaking, as soon as one of the components of $S_{n}$ reaches a new minimum, the running infimum is updated accordingly and therefore the corresponding component of $W_{n}$ is set to zero.

Proof. The proof follows by exploring all four possibilities, depending on the position of $W_{n}-X_{n+1}=S_{n+1}-\bigwedge_{i=0}^{n} S_{i}$ relative to the origin. For example, if $W_{n}-X_{n+1}$ is in the second quadrant, that is, if $S_{n+1}^{(1)} \leq \inf _{i \leq n} S_{i}^{(1)}$ and $S_{n+1}^{(2)} \geq \inf _{i \leq n} S_{i}^{(2)}$, then

$S_{n+1}^{(1)}=\inf _{i \leq n+1} S_{i}^{(1)}$, and $S_{n+1}^{(2)} \geq \inf _{i \leq n+1} S_{i}^{(2)}$.

Therefore $W_{n+1}=S_{n+1}-\bigwedge_{i=0}^{n+1} S_{i}=\left(0, S_{n+1}^{(2)}-\inf _{i \leq n+1} S_{i}^{(2)}\right)$, and remark that this is the same as $\left(W_{n}-X_{n+1}\right) \vee 0$. The other cases follow by analogous considerations, which completes the proof.

We can regard the finite horizon survival function $F_{n}^{s}\left(u^{(1)}, u^{(2)}\right):=\mathbb{P}\left(\tau_{\wedge}\left(u^{(1)}, u^{(2)}\right)>t_{n}\right)$

as the c.d.f. of a survival measure. Relation (3.2), Lemma 1 and Proposition 1 imply that this survival measure is nothing else but the distribution of the reflected random walk $W_{n}$ inside the nonnegative quadrant of $\mathbb{R}^{2}$.

Theorem 1 (Duality). The following identity relates the distribution of the reflected version of the random walk to the finite horizon survival functions of the reserve process:

$$
\begin{aligned}
& \mathbb{P}\left(R_{t_{i}} \succeq 0, i=1, \ldots, n \mid R_{0}=\left(u^{(1)}, u^{(2)}\right)\right) \\
& \quad=\mathbb{P}\left(W_{n} \preceq\left(u^{(1)}, u^{(2)}\right) \mid W_{0}=0\right) .
\end{aligned}
$$

Proof. That $W_{n}$ is the reflected version of the random walk follows directly from the fact that it is the solution of the recursive equation in Proposition 1. In view of Lemma 1 and (3.2), the other statement is obvious, so this concludes the proof.

Remark 3. In fact $\left\{W_{n}\right\}_{n \geq 0}$ is the waiting time process in an initially empty two-dimensional queueing model with two servers, each with its own queue, and with simultaneous arrivals and the same $\left\{A_{n}, B_{n}^{(1)}, B_{n}^{(2)}\right\}_{n \geq 0}$ input process as for the risk reserve process; $A_{n}$ indicates interarrival time and $\left(B_{n}^{(1)}, B_{n}^{(2)}\right)$ denotes the vector of service requirements for the two servers.

One-dimensional instances of the above 'duality relation' are well known in the risk insurance/queueing literature, see for example Asmussen and Albrecher (2010, p. 45, p. 161 etc.).

\section{A functional equation}

In this section we consider the Laplace-Stieltjes transform of the survival function $F_{n}^{s}\left(u^{(1)}, u^{(2)}\right)$; Theorem 1 and (3.3) imply that this equals the transform of the bivariate waiting time for the $n$th customer:

$$
\begin{aligned}
& \mathbb{E} e^{-s_{1} W_{n}^{(1)}-s_{2} W_{n}^{(2)}}=\int e^{-s_{1} u^{(1)}-s_{2} u^{(2)}} \mathrm{d} F_{n}^{s}\left(u^{(1)}, u^{(2)}\right), \\
& \quad \mathcal{R e} s_{i} \geq 0, i=1,2 .
\end{aligned}
$$

Our main goal in this section is to obtain a recursion between the LSTs of $\left(W_{n+1}^{(1)}, W_{n+1}^{(2)}\right)$ and $\left(W_{n}^{(1)}, W_{n}^{(2)}\right)$ using the path-wise recursion in Proposition 1. But first we point out a sample-path relation between the reserve process and the reflected random 
walk which will be useful in the next section (the relation in Lemma 1 holds in distribution only). The event that any of the reserves is running at the maximum is the same as the event that the corresponding component of the reflected random walk is at 0 .

\section{Lemma 2.}

$$
\begin{aligned}
& \left\{W_{n+1}^{(i)}=0\right\} \\
& \quad=\left\{R_{t_{n+1}}^{(i)}=u^{(i)}+\max \left(0,-S_{1}^{(i)}, \ldots,-S_{n+1}^{(i)}\right)\right\}, \quad i=1,2 .
\end{aligned}
$$

Proof. $R_{t_{n+1}}^{(i)}=u^{(i)}-S_{n+1}^{(i)}$ and notice the following equivalence:

$$
\begin{aligned}
& S_{n+1}^{(i)}-\min \left(0, S_{1}^{(i)}, \ldots, S_{n}^{(i)}, S_{n+1}^{(i)}\right)=0 \\
& \quad \Leftrightarrow-S_{n+1}^{(i)}=\max \left(0,-S_{1}^{(i)}, \ldots,-S_{n}^{(i)},-S_{n+1}^{(i)}\right) .
\end{aligned}
$$

Remark 4. The event $\left\{W_{n}^{(i)}=0\right\}$ does not depend on the initial capital. The event on the RHS in Lemma 2 does not restrict the reserve process to staying above 0 ; equivalently $W_{n}^{(i)}$ on the LHS is not restricted to staying below level $u^{(i)}$. This is in line with (3.3) in Theorem 1.

Below we point out how one can obtain a recursive equation for the LST of the survival function $F_{n}^{s}\left(u^{(1)}, u^{(2)}\right)$ in the general case without the ordering assumption. In Section 5 we solve this equation for the case when the risks are ordered.

The bivariate recursion in Proposition 1 becomes in terms of LSTs:

$\mathbb{E} e^{-s_{1} W_{n+1}^{(1)}-s_{2} W_{n+1}^{(2)}}=\mathbb{E} e^{-s_{1}\left(W_{n}^{(1)}-X_{n+1}^{(1)}\right)^{+}-s_{2}\left(W_{n}^{(2)}-X_{n+1}^{(2)}\right)^{+}}$,

with $(x)^{+}$denoting $\max (x, 0)$. Hence

$$
\begin{aligned}
\mathbb{E} e^{-s_{1} W_{n+1}^{(1)}-s_{2} W_{n+1}^{(2)}} & \\
= & \mathbb{E}\left[e^{-s_{1}\left(W_{n}^{(1)}-X_{n+1}^{(1)}\right)-s_{2}\left(W_{n}^{(2)}-X_{n+1}^{(2)}\right)} 1_{\left\{X_{n+1}^{(1)}<W_{n}^{(1)}, X_{n+1}^{(2)}<W_{n}^{(2)}\right\}}\right] \\
+ & \mathbb{E}\left[e^{-s_{1}\left(W_{n}^{(1)}-X_{n+1}^{(1)}\right)} 1_{\left.\left\{X_{n+1}^{(1)}<W_{n}^{(1)}, X_{n+1}^{(2)} \geq W_{n}^{(2)}\right\}\right]}\right] \\
+ & \mathbb{E}\left[e^{-s_{2}\left(W_{n}^{(2)}-X_{n+1}^{(2)}\right)} 1_{\left\{X_{n+1}^{(1)} \geq W_{n}^{(1)}, X_{n+1}^{(2)}<W_{n}^{(2)}\right\}}\right] \\
& +\mathbb{P}\left(X_{n+1}^{(1)} \geq W_{n}^{(1)}, X_{n+1}^{(2)} \geq W_{n}^{(2)}\right) .
\end{aligned}
$$

In view of (4.1), the left-hand side of (4.2) represents the LST of the survival measure $F_{n+1}^{s}$. Below we also interpret each of the four terms in the right hand side in terms of transforms of survival measures.

Term 1: In terms of excursions away from the maximum reserve (Lemma 2), the first term on the RHS represents the transform of the survival measure in the event that both reserves are during an excursion below the running maximum at time $t_{n+1}$,

$$
\begin{aligned}
& \mathbb{E} e^{-s_{1} W_{n+1}^{(1)}-s_{2} W_{n+1}^{(2)}} 1_{\left\{W_{n+1}^{(1)}>0, W_{n+1}^{(2)}>0\right\}} \\
& =\int e^{-s_{1} u^{(1)}-s_{2} u^{(2)}} 1_{\left\{R_{t_{n+1}} \prec\right.} \underset{k=0, \ldots, n}{\bigvee} R_{\left.t_{k}\right\}} \mathrm{d} F_{n+1}^{s}\left(u^{(1)}, u^{(2)}\right) .
\end{aligned}
$$

Above we used that on the event $\left\{W_{n+1}^{(i)}>0\right\}$, it holds that $W_{n}^{(i)}-$ $X_{n+1}^{(i)}=W_{n+1}^{(i)}$ (Proposition 1).

Terms 2 and 3: These can be translated in terms of survival functions using (4.1) again:

$$
\begin{aligned}
& \mathbb{E} e^{-s_{1} W_{n+1}^{(1)}} 1_{\left\{W_{n+1}^{(1)}>0, W_{n+1}^{(2)}=0\right\}} \\
& =\int_{0+}^{\infty} e^{-s_{1} u^{(1)}} 1_{\left\{R_{t_{n+1}}^{(1)}<\max _{k=0, \ldots, n} R_{t_{k}}^{(1)} ; R_{t_{n+1}}^{(2)} \geq_{k=0, \ldots, n} \max _{t_{k}} R^{(2)} \mathrm{d} F_{n+1}^{s}\left(u^{(1)}, 0\right),\right.} \\
& \mathbb{E} e^{-s_{2} W_{n+1}^{(2)}} 1_{\left\{W_{n+1}^{(1)}=0, W_{n+1}^{(2)}>0\right\}} \\
& =\int_{0+}^{\infty} e^{-s_{2} u^{(2)}} 1_{\left\{R_{t_{n+1}}^{(1)} \geq_{k=0, \ldots, n} \max _{t_{k}} ; R_{t_{n+1}}^{(2)}<\max _{k=0, \ldots, n} R_{t_{k}}^{(2)}\right\}} \mathrm{d} F_{n+1}^{s}\left(0, u^{(2)}\right),
\end{aligned}
$$

are 'boundary' transforms. The survival measure that corresponds to $F_{n}^{s}$ can have positive mass on the axes of the non-negative quadrant, if nowhere else, at least $F_{n}^{s}(0,0)=\mathbb{P}\left(W_{n}=0 \mid W_{0}=0\right)$ is positive, i.e., it has an atom in the origin.

Term 4: This is the probability that both reserves are surviving and running at a maximum, which by Lemma 2 and Theorem 1 is $F_{n+1}^{s}(0,0)$.

From an analytic point of view it is more convenient to rewrite (4.2) as a recursion. After adding and subtracting appropriate terms, one obtains,

$$
\begin{aligned}
& \mathbb{E} e^{-s_{1} W_{n+1}^{(1)}-s_{2} W_{n+1}^{(2)}}=\mathbb{E} e^{s_{1} X_{n+1}^{(1)}+s_{2} X_{n+1}^{(2)}} \mathbb{E} e^{-s_{1} W_{n}^{(1)}-s_{2} W_{n}^{(2)}} \\
& +\mathbb{E}\left\{e^{-s_{1}\left(W_{n}^{(1)}-X_{n+1}^{(1)}\right)}\left[1-e^{-s_{2}\left(W_{n}^{(2)}-X_{n+1}^{(2)}\right)}\right] 1_{\left\{W_{n+1}^{(1)}>0, W_{n+1}^{(2)}=0\right\}}\right\} \\
& +\mathbb{E}\left\{e^{-s_{2}\left(W_{n}^{(2)}-X_{n+1}^{(2)}\left[1-e^{-s_{1}\left(W_{n}^{(1)}-X_{n+1}^{(1)}\right)}\right] 1_{\left\{W_{n+1}^{(1)}=0, W_{n+1}^{(2)}>0\right\}}\right\}}\right. \\
& +\mathbb{E}\left\{\left[1-e^{-s_{1}\left(W_{n}^{(1)}-X_{n+1}^{(1)}\right)-s_{2}\left(W_{n}^{(2)}-X_{n+1}^{(2)}\right)}\right] 1_{\left\{W_{n+1}^{(1)}=0, W_{n+1}^{(2)}=0\right\}}\right\} .
\end{aligned}
$$

Above we used that the increment $X_{n+1}$ is independent of $W_{n}$. Under the assumption that the vector $W_{n}$ has a limit in distribution, $W$, as $n \rightarrow \infty$, (4.3) becomes

$$
\begin{aligned}
& K\left(s_{1}, s_{2}\right) \mathbb{E} e^{-s_{1} W^{(1)}-s_{2} W^{(2)}} \\
& =\mathbb{E}\left\{e^{-s_{1}\left(W^{(1)}-X^{(1)}\right)}\left[1-e^{-s_{2}\left(W^{(2)}-X^{(2)}\right)}\right] 1_{\left\{W^{(1)}>X^{(1)}, W^{(2)} \leq X^{(2)}\right\}}\right\} \\
& \quad+\mathbb{E}\left\{e^{-s_{2}\left(W^{(2)}-X^{(2)}\right)}\left[1-e^{-s_{1}\left(W^{(1)}-X^{(1)}\right)}\right] 1_{\left\{W^{(1)} \leq X^{(1)}, W^{(2)}>X^{(2)}\right\}}\right\} \\
& \quad+\mathbb{E}\left\{\left[1-e^{-s_{1}\left(W^{(1)}-X^{(1)}\right)-s_{2}\left(W^{(2)}-X^{(2)}\right)}\right] 1_{\left\{W^{(1)} \leq X^{(1)}, W^{(2)} \leq X^{(2)}\right\}}\right\},
\end{aligned}
$$

with "kernel" $K\left(s_{1}, s_{2}\right):=1-\mathbb{E} e^{s_{1} X^{(1)}+s_{2} X^{(2)}}$ and $\mathcal{R e} s_{i}=0, i=$ $1,2$.

Remark 5. From Lemma $1, W$ has the same distribution as the alltime supremum $M:=\lim _{n \rightarrow \infty} M_{n}$; and $M_{n}$ being a sequence of nondecreasing random vectors w.r.t. the order ' $\preceq$ ', the limit always exists a.s., although it may have a defective distribution. In the next section we give a sufficient condition for $M$ to have a proper distribution under the assumption that risks are ordered.

\section{Wiener-Hopf analysis of the stochastic recursion}

In this section we resolve the functional equation (4.4) under Assumptions 2.1 and 2.2, i.e., we find the LST of the infinite horizon survival function:

$F^{s}=\lim _{n \rightarrow \infty} F_{n}^{s}$,

the limit being considered in distribution. Theorem 1 together with a limit argument shows that this weak limit is the same as the c.d.f. of the stationary version of the waiting time process $\left(W_{n}\right)_{n \geq 0}$ (see also Remark 3).

The section is divided into three subsections. Section 5.1 prepares the ground, by making a key observation about the functional equation (4.4), introducing some notation and discussing the stability condition. Section 5.2 expresses the two-dimensional LST $\psi\left(s_{1}, s_{2}\right)$ of $F^{s}$ in a one-dimensional unknown function $C\left(s_{1}\right)$ (Proposition 2). That function is determined in Section 5.3, yielding our main result: Theorem 2 . 


\subsection{Preparations}

Introduce the extra claim amount $D_{n}:=B_{n}^{(1)} / c^{(1)}-B_{n}^{(2)} / c^{(2)}=$ $X_{n}^{(2)}-X_{n}^{(1)}$, so that the increments of the random walk $S_{n}$ can be represented as $-X_{n}=\left(-X_{n}^{(2)}+D_{n},-X_{n}^{(2)}\right)$.

We first make the following key observation: The ordering assumption (2.4) implies that when $R_{t_{n}}^{(1)}$ is at a maximum, necessarily $R_{t_{n}}^{(2)}$ is at a maximum. Via Lemma 2, this corresponds to the fact that the events

$\left\{W_{n}^{(1)} \leq X_{n+1}^{(1)}, W_{n}^{(2)}>X_{n+1}^{(2)}\right\}=\left\{W_{n+1}^{(1)}=0, W_{n+1}^{(2)}>0\right\}$

are null for all $n \geq 0$. This means that the third term on the RHS of (4.3) is null and hence the second term on the RHS of (4.4) vanishes as well, so that after regrouping terms, (4.4) can be rewritten as

$K\left(s_{1}, s_{2}\right) \psi\left(s_{1}, s_{2}\right)=-\psi_{1}\left(s_{1}, s_{2}\right)+\psi_{2}\left(s_{1}\right)+\mathbb{P}\left(X^{(1)} \geq W^{(1)}\right),(5.1)$

where

$\psi_{1}\left(s_{1}, s_{2}\right)=\mathbb{E} e^{-s_{1}\left(W^{(1)}-X^{(2)}+D\right)-s_{2}\left(W^{(2)}-X^{(2)}\right)} 1_{\left\{X^{(2)} \geq W^{(2)}\right\}}$,

$\psi_{2}\left(s_{1}\right)=\mathbb{E} e^{-s_{1}\left(W^{(1)}-X^{(2)}+D\right)} 1_{\left\{W^{(1)}+D>X^{(2)} \geq W^{(2)}\right\}}$.

Consider the following function:

$\tilde{K}\left(s_{1}, z\right):=1-\mathbb{E} e^{-s_{1} D+z X^{(2)}}, \quad$ Re $s_{1} \geq 0$, Re $z=0$.

This is related to $K\left(s_{1}, s_{2}\right)$ that appears in (4.4) through a change of coordinates: $\tilde{K}\left(s_{1}, z\right)=K\left(s_{1}, z-s_{1}\right)$. In addition, remark that for fixed $z, \tilde{K}\left(s_{1}, z\right)$ is indeed analytic in $\mathcal{R} e s_{1}>0$ because $D \geq 0$ a.s. Now let us change the coordinates: $\left(s_{1}, s_{2}\right) \rightarrow\left(s_{1}, s_{1}+s_{2}\right)=$ : $\left(s_{1}, z\right)$, and denote $\tilde{\psi}\left(s_{1}, z\right):=\psi\left(s_{1}, z-s_{1}\right)$. Then $\psi_{1}\left(s_{1}, s_{2}\right)$ becomes

$$
\begin{aligned}
\psi_{1}\left(s_{1}, s_{2}\right) & =\mathbb{E}\left[e^{-s_{1}\left(W^{(1)}-W^{(2)}+D\right)-z\left(W^{(2)}-X^{(2)}\right)} 1_{\left\{X^{(2)} \geq W^{(2)}\right\}}\right] \\
& =: \tilde{\psi}_{1}\left(s_{1}, z\right),
\end{aligned}
$$

and therefore (5.1) can be rewritten as

$\tilde{K}\left(s_{1}, z\right) \tilde{\psi}\left(s_{1}, z\right)=-\tilde{\psi}_{1}\left(s_{1}, z\right)+\psi_{2}\left(s_{1}\right)+\mathbb{P}\left(X^{(1)} \geq W^{(1)}\right)$.

Running example: One of the simplest examples that we will use throughout is obtained when taking the joint distribution of $\left(A, D, B^{(2)}\right)$ to be such that conditional on a random variable $N$, these $A, D$ and $B^{(2)}$ are independent and have Erlang distributions of order $N$ and rates respectively $\lambda, \mu_{D}$ and $\mu$. To keep things as simple as possible, we choose $\mathbb{P}(N=1)=\mathbb{P}(N=2)=\frac{1}{2}$ and rates $\lambda=1, \mu_{D}=3, \mu=2$; we also choose the income rates $c^{(1)}$ and $c^{(2)}$ to be equal to 1 .

The kernel $\tilde{K}\left(s_{1}, z\right)$ has the following simple form:

$\tilde{K}\left(s_{1}, z\right)=1-\frac{3\left(3+s_{1}\right)(1-z)(2+z)+18}{\left(3+s_{1}\right)^{2}(1-z)^{2}(2+z)^{2}}$

From a specific example like this it becomes clear that the coefficients of the numerator, for example, as a polynomial in $z$ are themselves polynomials in $s_{1}$ and vice versa.

We are now ready to formulate a Wiener-Hopf boundary value problem in variable $z$. For fixed $s_{1}$, Re $s_{1}>0, \tilde{\psi}_{1}\left(s_{1}, z\right)$ is analytic in $\mathcal{R} e z<0$ (by analytic continuation), while $\tilde{\psi}\left(s_{1}, z\right)$ is analytic (by analytic continuation) in $\mathcal{R e} z>0$. These statements follow easily from the probabilistic nature of these functions. In particular, notice that $\tilde{\psi}\left(s_{1}, z\right)=\mathbb{E} \mathrm{e}^{-s_{1}\left(W^{(1)}-W^{(2)}\right)-z W^{(2)}}$. Also on the event $\left\{W^{(2)}<X^{(2)}\right\}$, the random variable $e^{-z\left(W^{(2)}-X^{(2)}\right)}$ is uniformly bounded in $\operatorname{Re} z \leq 0$, hence the analyticity of $\tilde{\psi}_{1}\left(s_{1}, z\right)$ follows by an application of Lebesgue's dominated convergence theorem.

The approach we take in order to solve (5.2) uses a Wiener-Hopf factorization with a parameter. More precisely, for each fixed $s_{1}, \mathcal{R} e s_{1}>0$, we will factorize the bivariate kernel $\tilde{K}\left(s_{1}, z\right)=$ $\tilde{K}_{s_{1}}(z)$ that appears in (5.2) into $\tilde{K}_{s_{1}}(z)=\tilde{K}_{s_{1}}^{+}(z) \tilde{K}_{s_{1}}^{-}(z)$, such that $\tilde{K}_{s_{1}}^{+}(z)$ can be analytically continued in $\mathcal{R} e z>0$ and $\tilde{K}_{s_{1}}^{-}(z)$ can be analytically continued in $\mathcal{R} e z<0$. The Wiener-Hopf factorization that solves (5.2) is discussed in the next subsection. Finally, once we resolve (5.2), the solution to (5.1) follows by reverting to the original coordinate system $\left(s_{1}, s_{2}\right)$.

Remark 6. A reason why we prefer the notation using the argument $s_{1}$ as a subscript $\tilde{K}_{s_{1}}^{ \pm}(z)$ for these factors is that they are in general obtained by pasting together different branches of multivalued complex functions in $s_{1}$ using analytic continuation. More precisely, since $\tilde{K}\left(s_{1}, z\right)$ is a rational function, the 1-parameter Wiener-Hopf factors may have branch cuts in $\mathcal{R} e s_{1}>0$ (discontinuities) as functions of the argument $s_{1}$; then as it follows from Proposition 2, we must choose the values of the zeros of the kernel that have positive real part for $\mathcal{R e} s_{1}>0$ and glue them together (using analytic continuation). Because of this, the 1-parameter Wiener-Hopf factors $\tilde{K}_{s_{1}}^{+}(z)$ and $\tilde{K}_{s_{1}}^{-}(z)$ are not functions of $s_{1}$ in the real sense. This will be the case with the zeros of the kernel from Example 2 in Section 6.

Finally a word about conditions under which the limiting distribution of the two-dimensional waiting time process $\left\{W_{n}^{(1)}, W_{n}^{(2)}\right\}_{n=1,2, \ldots}$ exists, or equivalently, under which survival of both risk reserves has a positive probability. It will turn out from the analysis below that a necessary condition for the existence of a proper limit in distribution $W$ is $\rho_{1}:=\mathbb{E} B^{(1)} /\left(c^{(1)} \mathbb{E} A\right)<1$. This is easy to interpret in our case, because it is sufficient to ensure positive safety loading for line 1 which receives always larger claims - we then automatically have positive safety loading for the second insurance line. The safety loading condition for the second reserve process $\rho_{2}:=\mathbb{E} B^{(2)} /\left(c^{(2)} \mathbb{E} A\right)<1$ will be necessary for the Wiener-Hopf factorization to hold. The two Wiener-Hopf factors will be initially determined up to a certain unknown 'boundary' function $C\left(s_{1}\right)$ that appears in Eq. (5.4); we further determine this boundary function by noting that the marginal reserve process $R_{t}^{(1)}$ behaves as a (one-dimensional) generalized Sparre-Andersen reserve process with dependence between inter-arrival times and subsequent claim sizes, for which an analysis of the survival function is available in Badila et al. (2013). At this point the safety loading condition $\rho_{1}<1$ becomes necessary.

\subsection{A Wiener-Hopf factorization}

In this subsection we determine the double transform $\tilde{\psi}\left(s_{1}, z\right)$ up to a - yet - unknown one-dimensional function $C\left(s_{1}\right)$. In the next subsection we will determine this function, which turns out to be related to the first insurance line only.

Proposition 2 (Wiener-Hopf Factorization with a Parameter). Under Assumption 2.1 the double LST $\tilde{\psi}\left(s_{1}, z\right)$ is of the form

$\tilde{\psi}\left(s_{1}, z\right)=\mathbb{E} e^{-s_{1}\left(W^{(1)}-W^{(2)}\right)-z W^{(2)}}=C\left(s_{1}\right) \tilde{K}_{s_{1}}^{+}(z)^{-1}$.

$C\left(s_{1}\right)$ is a yet to be determined analytic function, $\mathcal{R} e s_{1}>0$. For fixed $\operatorname{Re} s_{1}>0, \tilde{K}_{s_{1}}^{+}(z)$ is analytic for $\operatorname{Re} z>0$, continuous up to the boundary and it factorizes $\tilde{K}\left(s_{1}, z\right)$ into

$\tilde{K}\left(s_{1}, z\right)=\tilde{K}_{s_{1}}^{+}(z) \tilde{K}_{s_{1}}^{-}(z)$

such that $\tilde{K}_{s_{1}}^{-}(z)$ is analytic for $\mathcal{R e} z<0$ and continuous up to the boundary.

Proof. Under Assumption 2.1, the triple transform of $A, B^{(1)} / c^{(1)}$, $B^{(2)} / c^{(2)}$ is $H\left(q_{0}, q_{1} / c^{(1)}, q_{2} / c^{(2)}\right)$ and the kernel is $\tilde{K}\left(s_{1}, z\right)=1$ 
$-H\left(-z, s_{1} / c^{(1)},\left(z-s_{1}\right) / c^{(2)}\right)$ which is also a rational function with representation $\tilde{K}\left(s_{1}, z\right):=1-\frac{f\left(s_{1}, z\right)}{g\left(s_{1}, z\right)}$. The assumption that $A>0$ a.s. implies that the degree of $f_{s_{1}}(z)$ is strictly less than the degree of $g_{s_{1}}(z)$ as polynomial functions in the argument $z$ (see the discussion in Remark 6). Now the functional equation (5.2) becomes

$$
\begin{aligned}
& \frac{g\left(s_{1}, z\right)-f\left(s_{1}, z\right)}{g\left(s_{1}, z\right)} \tilde{\psi}\left(s_{1}, z\right) \\
& =-\tilde{\psi}_{1}\left(s_{1}, z\right)+\psi_{2}\left(s_{1}\right)+\mathbb{P}\left(X^{(1)} \geq W^{(1)}\right) .
\end{aligned}
$$

The first step is to factorize the kernel into two factors with respect to the $z$ variable and regroup (5.5) into an analytic function in $\operatorname{Re} z>0$ on the LHS and an analytic function in $\mathcal{R} e z<0$ on the RHS. Once we have this representation, we can use Liouville's Theorem to determine both sides of (5.5) up to the function $C\left(s_{1}\right)$.

Remove all the poles with nonnegative real part from the LHS of (5.5). We keep for now $s_{1}$ fixed with $\mathcal{R} e s_{1} \geq 0$, and denote by

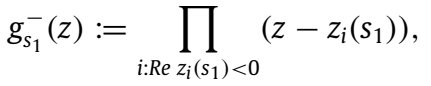

where $z_{i}\left(s_{1}\right)$ are zeros of $g\left(s_{1}, z\right)=g_{s_{1}}(z)$; also put $g_{s_{1}}^{+}(z):=$ $\frac{g_{s_{1}}(z)}{g_{s_{1}}^{-}(z)}$, so that we have the factorization $g_{s_{1}}(z)=g_{s_{1}}^{+}(z) g_{s_{1}}^{-}(z)$. Upon multiplying both sides of (5.5) by $g_{s_{1}}^{+}(z)$, the LHS becomes analytic for all $\operatorname{Re} z>0$ and continuous up to the imaginary axis. Similarly, the RHS is analytic for all $\mathcal{R} e z<0$ and continuous for $\mathcal{R e} z \leq 0$. Since these two coincide for $\mathcal{R e} z=0$, they are analytic continuations of each other, in particular $\frac{g_{s_{1}}(z)-f_{s_{1}}(z)}{g_{s_{1}}^{-}(z)} \tilde{\psi}_{s_{1}}(z)$ is an entire function in $z$. Because $\operatorname{deg} f_{s_{1}}(z) \leq \operatorname{deg} g_{s_{1}}(z)$, asymptotically $\frac{g_{s_{1}}(z)-f_{s_{1}}(z)}{g_{s_{1}}^{-}(z)} \tilde{\psi}_{s_{1}}(z)=O\left(z^{m_{+}\left(s_{1}\right)}\right)$,

where $m_{+}\left(s_{1}\right):=\operatorname{deg} g_{s_{1}}^{+}(z)$. By virtue of Liouville's theorem (Titchmarsh, 1939, p. 85),

$\tilde{\psi}_{s_{1}}(z)=\frac{g_{s_{1}}^{-}(z)}{g_{s_{1}}(z)-f_{s_{1}}(z)} P_{s_{1}}(z)$,

where (for fixed $s_{1} \geq 0$ ), $P_{s_{1}}(z)$ is a polynomial in $z$ with $\operatorname{deg} P_{s_{1}}(z)$ $\leq m_{+}\left(s_{1}\right)$. From (5.6) it follows immediately that $P_{s_{1}}(z)$ must have all the zeros with non-negative real part of the denominator $g_{s_{1}}(z)-f_{s_{1}}(z)$. Now a key part in the argument is the fact that the denominator $g_{s_{1}}(z)-f_{s_{1}}(z)$ has the same number of zeros in $\operatorname{Re} z \geq 0$ as $g_{s_{1}}^{+}(z)$. The proof of this statement is deferred to the Appendix in Proposition A.1. Thus we have $\operatorname{deg} P_{s_{1}}(z) \geq m_{+}$. Together with the upper bound on the degree of $P_{s_{1}}(z)$, this implies $\operatorname{deg} P_{s_{1}}(z)=m_{+}$; moreover, this determines $P_{s_{1}}(z)$ up to a constant factor (constant being relative to $z$ !)

$P_{s_{1}}(z)=C\left(s_{1}\right) \prod_{i: \mathcal{R e} v_{i}\left(s_{1}\right) \geq 0}\left(z-v_{i}\left(s_{1}\right)\right)$,

where $v_{i}\left(s_{1}\right)$ are zeros of $g_{s_{1}}(z)-f_{s_{1}}(z)$. Upon replacing the above in (5.6), we have found the one-parameter positive Wiener-Hopf factor

$\tilde{K}_{s_{1}}^{+}(z)=\frac{\prod_{i: \mathcal{R} e v_{i}\left(s_{1}\right)<0}\left(z-v_{i}\left(s_{1}\right)\right)}{g_{s_{1}}^{-}(z)}$.

And in particular the above also determines $\tilde{K}_{s_{1}}^{-}(z)$ :

$\tilde{K}_{s_{1}}^{-}(z)=\frac{\tilde{K}\left(s_{1}, z\right)}{\tilde{K}_{s_{1}}^{+}(z)}$,

and the proof is complete.
Running example: For fixed $s_{1}$, we can carry out the factorization for the kernel (5.3) in the running example. Below we give the zeros of the numerator

$$
\begin{aligned}
& v_{1}\left(s_{1}\right)=\frac{-s_{1}-3-\sqrt{3} \sqrt{\left(s_{1}+3\right)\left(1+3 s_{1}\right)}}{2\left(3+s_{1}\right)}, \\
& v_{2}\left(s_{1}\right)=\frac{-s_{1}-3+\sqrt{3} \sqrt{\left(s_{1}+3\right)\left(3 s_{1}+1\right)}}{2\left(3+s_{1}\right)}, \\
& v_{3}\left(s_{1}\right)=\frac{-s_{1}-3-\sqrt{\left(s_{1}+3\right)\left(3 s_{1}+13\right)}}{2\left(3+s_{1}\right)}, \\
& v_{4}\left(s_{1}\right)=\frac{-s_{1}-3+\sqrt{\left(s_{1}+3\right)\left(3 s_{1}+13\right)}}{2\left(3+s_{1}\right)} .
\end{aligned}
$$

The radicals above are defined when the cut in the complex plane is taken along the negative half of the real axis and the complex arguments are measured from $-\pi$ to $\pi$. This convention determines the so-called principal value of the square root function. The negative real half-axis will be a discontinuity line for the square root function $\sqrt{z}$ as a function of a complex variable because as the argument $z$ approaches the negative real half-axis,

$\left(z^{-}-z_{0}\right)^{1 / 2}=e^{i \pi}\left(z^{+}-z_{0}\right)^{1 / 2}$,

where $z_{0}$ lies on the negative real half-axis, that is, $\mathcal{R} e z_{0}<0$ and $\operatorname{lm} z_{0}=0 ; z^{ \pm}$denotes the limit of $z$ towards $z_{0}$ from respectively above and below the real axis. We will call such lines of discontinuity branch cuts. The branch points of $v_{1}$ (and $v_{2}$ ) are -3 and $-1 / 3$. The branch cuts are then the curves generated by the equations

$\arg \left(s_{1}+3\right)+\arg \left(1+3 s_{1}\right)= \pm \pi$.

It is a problem of plane geometry to see that these branch cuts are constituted by the line segment joining the two branch points, together with the perpendicular line on this segment that passes through its mid-point. The situation is similar for $v_{3}\left(s_{1}\right)$ and $v_{4}\left(s_{1}\right)$.

By inspecting the zeros of the numerator for $\mathcal{R} e s_{1}>0$, exactly $v_{1}\left(s_{1}\right)$ and $v_{3}\left(s_{1}\right)$ are negative, where by positive/negative values of complex numbers we will always refer to their real parts. There are as many negative zeros in the denominator, which is already confirmed by Proposition A.1. Moreover, the branch cuts of neither $v_{1}$ nor $v_{3}$ are located in the right half-plane, which means these zeros are regular functions for positive values of $s_{1}$.

Having isolated the negative zeros, the one-parameter positive Wiener-Hopf factor from (5.7) is

$\tilde{K}_{s_{1}}^{+}(z)$

$$
=\frac{\left(z-\frac{-s_{1}-3-\sqrt{3} \sqrt{\left(s_{1}+3\right)\left(1+3 s_{1}\right)}}{2\left(s_{1}+3\right)}\right)\left(z-\frac{-s_{1}-3-\sqrt{\left(s_{1}+3\right)\left(3 s_{1}+13\right)}}{2\left(s_{1}+3\right)}\right)}{(z+2)^{2}} .
$$

Remark 7. Interestingly, $\tilde{K}_{s_{1}}^{+}$is not a rational function anymore in the argument $s_{1}$ (however, in this example it is meromorphic in the argument $s_{1}$, for $\mathcal{R} e s_{1}>0$ ). A queueing theoretic explanation of this remark can be found by comparing the double transform (5.9) obtained below with the decomposition results for a particular case of the present model in Badila et al. (2014). For the process with Markov arrivals studied therein, the stationary waiting time stochastically decomposes into two components, one of which is related to the extra busy period length in the longest queue and it is well known that busy periods in general do not have rational transforms (already in the case of an $M / M / 1$ system, the busy period has a non-rational transform).

Moreover, it is easy to see that the marginal factor $\left.\tilde{K}_{s_{1}}^{+}\left(s_{2}\right)\right|_{s_{1}=0}$ is a rational function. From this and (5.9) follows that the marginal transform $\psi\left(0, s_{2}\right)$ is a rational function. Also, $\psi\left(s_{1}, 0\right)$ is a rational function because for $s_{2}=0$ the factor $\tilde{K}_{s_{1}}^{+}$cancels against itself in 
(5.9). The rationality of the marginal transforms is clear from their queueing interpretation because these are the transforms of the univariate survival functions/waiting times for the two insurance lines/queueing systems in isolation (see the discussion in Cohen, 1982, p. 325 and the references therein).

\subsection{The main result}

We are now ready to formulate and prove the main result.

Theorem 2. Under the safety loading condition for the riskier line 1, $\rho_{1}<1$, the infinite horizon survival function $F^{s}\left(u^{(1)}, u^{(2)}\right)$ is $a$ (proper) probability distribution function with support the nonnegative quadrant in $\mathbb{R}^{2}$, and its LST is given by

$$
\begin{aligned}
\psi\left(s_{1}, s_{2}\right) & =\int e^{-s_{1} u^{(1)}-s_{2} u^{(2)}} \mathrm{d} F^{s}\left(u^{(1)}, u^{(2)}\right) \\
& =\frac{K_{p r}^{+}(0)}{K_{p r}^{+}\left(s_{1}\right)} \frac{\tilde{K}_{s_{1}}^{+}\left(s_{1}\right)}{\tilde{K}_{s_{1}}^{+}\left(s_{1}+s_{2}\right)},
\end{aligned}
$$

for $\mathcal{R} e s_{i} \geq 0, i=1,2 . \tilde{K}_{s_{1}}^{+}(z)$ is given in (5.7) and here is evaluated at $z=s_{1}$ and at $z=s_{1}+s_{2}$. $K_{p r}^{+}\left(s_{1}\right)$ is the positive Wiener-Hopffactor of the projected one-dimensional kernel $\tilde{K}\left(s_{1}, s_{1}\right)=K\left(s_{1}, 0\right)$, i.e., the unique function analytic in $\mathcal{R} e s_{1}>0$, continuous in $\mathcal{R e} s_{1} \geq 0$ that factorizes $K\left(s_{1}, 0\right)$ into

$K\left(s_{1}, 0\right)=K_{p r}^{+}\left(s_{1}\right) K_{p r}^{-}\left(s_{1}\right)$,

with $K_{p r}^{-}\left(s_{1}\right)$ analytic in $\mathcal{R e} s_{1}<0$ and continuous in $\mathcal{R e} s_{1} \leq 0$ (see for instance Prabhu, 1980, Thm. 7 p. 55). Under Assumption 2.1 it is of the form (see also Badila et al., 2013)

$$
K_{p r}^{+}\left(s_{1}\right)=\frac{\prod_{j}\left(s_{1}-\tilde{v}_{j}^{-}\right)}{\prod_{j}\left(s_{1}-v_{j}^{-}\right)},
$$

with $\tilde{v}_{j}^{-}$the negative zeros of $K\left(s_{1}, 0\right)$ and $v_{j}^{-}$its negative poles.

Proof. Our starting-point is (5.4), and our goal is to determine the one yet unknown function $C\left(s_{1}\right)$ in that formula. The idea is that, since $C\left(s_{1}\right)$ stays the same irrespective of the value of $z$, we are free to choose any $z$. Since by definition, $\tilde{K}_{s_{1}}^{+}(z)$ is analytic for all $\operatorname{Re} z>0$ and continuous in $\operatorname{Re} z \geq 0$, take $z=s_{1}$ :

$\tilde{\psi}\left(s_{1}, s_{1}\right)=\mathbb{E} e^{-s_{1} W^{(1)}}=C\left(s_{1}\right)\left[\tilde{K}_{s_{1}}^{+}\left(s_{1}\right)\right]^{-1}$.

We can determine $C\left(s_{1}\right)$ from (5.11), because $\tilde{\psi}\left(s_{1}, s_{1}\right)=$ $\psi\left(s_{1}, 0\right)=\mathbb{E} e^{-s_{1} W^{(1)}}$ is the steady-state waiting time transform in the marginal $G / G / 1$ queue with dependence between inter-arrival times and service requirements, which has been determined in Badila et al. (2013). That paper was devoted to an analysis of a one-dimensional risk/queueing model that amounts to the present model with $B^{(2)} \equiv 0$. The kernel of the functional identity for this marginal queue with generic service requirement $B^{(1)}$ and correlated inter-arrival time $A$ is $\tilde{K}\left(s_{1}, s_{1}\right)$; the corresponding Rouché problem is to prove that $g\left(s_{1}, s_{1}\right)$ and $g\left(s_{1}, s_{1}\right)-f\left(s_{1}, s_{1}\right)$ have the same number of nonnegative zeros. This has been carried out in Badila et al. (2013). Its Formula (6) reads

$\tilde{\psi}\left(s_{1}, s_{1}\right)=\mathbb{E} e^{-s_{1} W^{(1)}}=K_{p r}^{+}(0)\left[K_{p r}^{+}\left(s_{1}\right)\right]^{-1}$,

with $K_{p r}^{+}\left(s_{1}\right)$ the positive Wiener-Hopf factor of the projected kernel $K\left(s_{1}, 0\right)$ :

$K_{p r}^{+}\left(s_{1}\right)=\frac{\prod_{j}\left(s_{1}-\tilde{v}_{j}^{-}\right)}{\prod_{j}\left(s_{1}-v_{j}^{-}\right)}$, such that $\tilde{v}_{j}^{-}$are the negative zeros of $K\left(s_{1}, 0\right)$ and $v_{j}^{-}$its negative poles, and the normalizing constant $K_{p r}^{+}(0)$ is equal to the atom at 0 of $W^{(1)}$ :

$\mathbb{P}\left(W^{(1)}=0\right)=\prod_{k}\left(-v_{k}^{-}\right) / \prod_{j}\left(-\tilde{v}_{j}^{-}\right)$.

Formula (5.12) together with (5.11) now determine $C\left(s_{1}\right)$ :

$C\left(s_{1}\right)=K_{p r}^{+}(0)\left[K_{p r}^{+}\left(s_{1}\right)\right]^{-1} \tilde{K}_{s_{1}}^{+}\left(s_{1}\right)$,

and with this we obtain the transform of the joint waiting time distribution, or equivalently of the survival function $F^{s}\left(u^{(1)}, u^{(2)}\right)$, from (5.4), upon switching back to the original coordinates. The proof is complete.

Remark 8. An important remark is that the factors in (5.9), $\tilde{K}_{s_{1}}^{+}\left(s_{1}\right)$ and $K_{p r}^{+}\left(s_{1}\right)$, as defined in Proposition 2 and in Theorem 2, are not the same. One can already compare (5.8) with (6.2) for Example 1 in the following section.

More precisely, the operations of taking the projection and carrying out the factorization do not commute with each other, in contrast with the one-dimensional Fluctuation Theory of random walks. See also Section 13 in Kingman (1966).

$\tilde{K}_{s_{1}}^{+}\left(s_{1}\right)$ is defined by first carrying out the one-parameter Wiener-Hopf factorization for $\tilde{K}\left(s_{1}, z\right)$ and then projecting the positive factor onto the main diagonal of the 2-dimensional complex space: $z=s_{1}$.

On the other hand, $K_{p r}^{+}\left(s_{1}\right)$ is obtained by first projecting $\tilde{K}\left(s_{1}, z\right)$ onto the main diagonal $z=s_{1}$ and then carrying out the WienerHopf factorization for the projected kernel $\tilde{K}\left(s_{1}, s_{1}\right)=K\left(s_{1}, 0\right)$.

Remark 9. The LST in (5.9) has a product form. It can be shown that the bivariate LST of the reflected random walk decomposes in a similar way as in Theorem 1 of Badila et al. (2014), where one of the factors is related to a modified workload process.

\section{Examples and numerical inversion}

In the previous sections we dealt with some theoretical aspects related to obtaining the transform of the survival function. It turns out that additional insight can be obtained by applying the previous results to some specific examples. Our aims in this section are:

(i) to provide examples for which the Laplace-Stieltjes transforms of the survival measures can be calculated, based on the general results obtained in the previous section.

(ii) to explain the various analytic challenges that appear when one tries to determine the Laplace-Stieltjes transform of the survival measure for some specific classes of distributions for the input $\left(A, D, B^{(2)}\right)$.

(iii) numerical inversion of the bivariate Laplace-Stieltjes transform in (5.9) and the comparison between the risks for various possible correlations between the claim sizes $\left(B_{n}^{(1)}, B_{n}^{(2)}\right)$ and inter-arrival times $A_{n}$.

We begin by explaining the inversion algorithm and how we applied it. However, in order to obtain the input for the algorithm, we need to follow the steps in Section 5 and construct the Wiener-Hopf factors. It turns out that this presents a challenge because of the branch cuts (discontinuities) that the zeros of the kernel might have in the right half-plane of the complex $s_{1}$-plane. The running example from Section 5.1 can thus be considered a simple instance of the inversion algorithm.

Numerical inversion. For the purpose of inverting (5.9), consider the Laplace transform of the bivariate tail probability of the waiting 
time. By a straight-forward integration by parts, this can be related to the Laplace-Stieltjes transform of the waiting time/survival function:

$$
\begin{gathered}
\iint e^{-s_{1} u_{1}-s_{2} u_{2}} \mathbb{P}\left(W^{(1)}>u_{1}, W^{(2)}>u_{2}\right) \mathrm{d} u_{1} \mathrm{~d} u_{2} \\
=\frac{1}{s_{1} s_{2}}\left[1-\psi\left(s_{1}, 0\right)-\psi\left(0, s_{2}\right)+\psi\left(s_{1}, s_{2}\right)\right] .
\end{gathered}
$$

The key remark is that under mild conditions, this transform is continuous up to the boundary of the non-negative quadrant, as opposed to the Laplace transform of the survival function:

$\iint e^{-s_{1} u_{1}-s_{2} u_{2}} \mathbb{P}\left(W^{(1)} \leq u_{1}, W^{(2)} \leq u_{2}\right) \mathrm{d} u_{1} \mathrm{~d} u_{2}$

which has by definition a singularity at $\left(s_{1}, s_{2}\right)=(0,0)$. It is easy to see that for example

$$
\begin{aligned}
& \lim _{\substack{s_{1} \rightarrow 0 \\
\mathcal{R} e s_{1}>0}} \frac{1}{s_{1} s_{2}}\left[1-\psi\left(s_{1}, 0\right)-\psi\left(0, s_{2}\right)+\psi\left(s_{1}, s_{2}\right)\right] \\
& =\frac{1}{s_{2}}\left[\frac{\partial \psi}{\partial s_{1}}\left(0, s_{2}\right)-\frac{\partial \psi}{\partial s_{1}}(0,0)\right]
\end{aligned}
$$

and even further

$$
\begin{aligned}
& \lim _{\substack{s_{1}, s_{2} \rightarrow 0 \\
\mathcal{R} s_{1}, s_{2}>0}} \frac{1}{s_{1} s_{2}}\left[1-\psi\left(s_{1}, 0\right)-\psi\left(0, s_{2}\right)+\psi\left(s_{1}, s_{2}\right)\right] \\
& =\frac{\partial^{2} \psi}{\partial s_{1} \partial s_{2}}(0,0)
\end{aligned}
$$

and it is clear that this mixed derivative is equal to $\mathbb{E}\left[W^{(1)} W^{(2)}\right]$, which is the same as the left-hand side of (6.1) evaluated at $s_{1}=$ $s_{2}=0$. The partial derivatives on the right-hand side above must be considered as limits from the interior of the positive quadrant. The Laplace transform of the ruin function is continuous up to the boundary given that the above partial derivatives exist.

The main point of the above discussion is that we may now use the standard form of the multidimensional inversion algorithm developed in den Iseger (2006), for which it is essential that the Laplace transform is regular and continuous up to the boundary of the positive quadrant. The above trick of passing to tail probabilities thus frees one from considering modifications of the inversion algorithm for non-smooth functions (see den Iseger, 2006). Once the tail probability/ruin function has been obtained, the survival function follows from an identity similar to (6.1):

$$
\begin{aligned}
\mathbb{P}\left(W^{(1)}>x_{1}, W^{(2)}>x_{2}\right)= & 1-\mathbb{P}\left(W^{(1)} \leq x_{1}\right)-\mathbb{P}\left(W^{(2)} \leq x_{2}\right) \\
& +\mathbb{P}\left(W^{(1)} \leq x_{1}, W^{(2)} \leq x_{2}\right),
\end{aligned}
$$

for any $x_{1}, x_{2} \geq 0$.

There are no regularity problems with the transforms we will be working with throughout this section because they are all meromorphic in both arguments for positive real values (in some cases they are constructed from branches of various locally meromorphic functions via analytic continuation-see Example 2).

Above we discussed how to consider the input for the inversion algorithm; some remarks are also needed about the output. This is an $M_{1} \times M_{2}$ matrix, that represents the values of the ruin function $\mathbb{P}\left(\tau_{\vee}(\cdot, \cdot)<\infty\right)$ on a grid: the entry $(k, l)$ stands for

$$
\begin{aligned}
& \mathbb{P}\left(\tau_{\vee}\left((k-1) \Delta_{1},(l-1) \Delta_{2}\right)<\infty\right) \\
& \quad=\mathbb{P}\left(W^{(1)}>(k-1) \Delta_{1}, W^{(2)}>(l-1) \Delta_{2}\right),
\end{aligned}
$$

where $\Delta_{i}$ are division sizes. The values of the inverted transform are plotted in the figures below for various examples.
Example 1. This is the running example that started at (5.3). We have calculated the one-parameter Wiener-Hopf factor for this example in (5.8). For the LST of the survival function we need also the positive Wiener-Hopf factor of the projected kernel:

$\tilde{K}\left(s_{1}, s_{1}\right)=K\left(s_{1}, 0\right)=\frac{-9 s_{1}-35 s_{1}^{2}-s_{1}^{3}+18 s_{1}^{4}+8 s_{1}^{5}+s_{1}^{6}}{\left(-1+s_{1}\right)^{2}\left(2+s_{1}\right)^{2}\left(3+s_{1}\right)^{2}}$.

The numerator can be factorized as $s_{1}\left(s_{1}^{2}+4 s_{1}+1\right)\left(s_{1}^{3}+4 s_{1}^{2}+s_{1}-9\right)$ where the order 2 polynomial further factorizes as

$s_{1}^{2}+4 s_{1}+1=\left(s_{1}+2-\sqrt{3}\right)\left(s_{1}+2+\sqrt{3}\right)$

and the zeros of the factor $s_{1}^{3}+4 s_{1}^{2}+s_{1}-9$ are

$$
\begin{aligned}
v_{0}:= & -\frac{4}{3}+\frac{1}{3 \sqrt[3]{2}}(151-9 \sqrt{173})^{1 / 3} \\
& +\frac{1}{3 \sqrt[3]{2}}(151+9 \sqrt{173})^{1 / 3}, \\
v_{1}:= & -\frac{4}{3}-\frac{1}{6 \sqrt[3]{2}}(1+i \sqrt{3})(151-9 \sqrt{173})^{1 / 3} \\
& -\frac{1}{6 \sqrt[3]{2}}(1-i \sqrt{3})(151+9 \sqrt{173})^{1 / 3}, \\
v_{2}= & -\frac{4}{3}-\frac{1}{6 \sqrt[3]{2}}(1-i \sqrt{3})(151-9 \sqrt{173})^{1 / 3} \\
& -\frac{1}{6 \sqrt[3]{2}}(1+i \sqrt{3})(151+9 \sqrt{173})^{1 / 3}, \quad \text { with } v_{2}=\bar{v}_{1} .
\end{aligned}
$$

The positive Wiener-Hopf factor of the projected kernel becomes, cf. (5.10),

$K_{p r}^{+}\left(s_{1}\right)=\frac{\left(s_{1}^{2}+4 s_{1}+1\right)\left(s_{1}-v_{1}\right)\left(s_{1}-\bar{v}_{1}\right)}{\left(s_{1}+2\right)^{2}\left(s_{1}+3\right)^{2}}$.

(5.8) and (6.2) are the necessary components for constructing the LST of the survival function in this example, as given by (5.9). The atom at $(0,0)$ is

$$
\begin{aligned}
\mathbb{P}\left(W^{(1)}=0, W^{(2)}=0\right) & =\mathbb{P}\left(W^{(1)}=0\right)=K_{p r}^{+}(0) \\
& =\frac{\left|v_{1}\right|^{2}}{36} \approx 0.204 .
\end{aligned}
$$

Here we used the ordering between $W^{(1)}$ and $W^{(2)}$. Eq. (6.3) in Box I shows the final formula for the transform of the survival function in the original coordinates: $\sqrt{s_{1}+3}$ cannot be simplified. The reason is the same as the one given in Remark 11 below. The marginal transforms are

$$
\begin{aligned}
& \psi\left(s_{1}, 0\right)=\frac{\left|v_{1}\right|^{2}}{36} \frac{\left(2+s_{1}\right)^{2}\left(3+s_{1}\right)^{2}}{\left(s_{1}^{2}+4 s_{1}+1\right)\left(s_{1}-v_{1}\right)\left(s_{1}-\bar{v}_{1}\right)}, \\
& \psi\left(0, s_{2}\right)=\frac{(3+\sqrt{39})\left(s_{2}+2\right)^{2}}{4\left(6 s_{2}+3+\sqrt{39}\right)\left(s_{2}+1\right)} .
\end{aligned}
$$

The atom at zero of $W^{(2)}$ is approximately 0.575 . We have carried out the numerical inversion for the transform in Example 1: the division size is chosen $\Delta_{1}=\Delta_{2}=0.1$ and the grid size is $M_{1}=$ $M_{2}=2^{6}$. An important performance measure is the $5 \%$ quantile curve of the tail probability-the two dimensional version of the $5 \%$ quantile, also known as value at risk. This is the (not necessarily continuous in general) curve that contains all $\left(u_{1}, u_{2}\right)$, such that $\mathbb{P}\left(W^{(1)}>u_{1}, W^{(2)}>u_{2}\right) \geq 0.05$ and $\mathbb{P}\left(W^{(1)}>u_{1}+, W^{(2)}>\right.$ $\left.u_{2}+\right) \leq 0.05$. To put it simply, the ruin function $\mathbb{P}\left(\tau_{\vee}(\cdot, \cdot)<\infty\right)$ (see Formula (2.2) and Remark 2) is less than $5 \%$ whenever it is evaluated at a point which lies outside the region bounded by this curve in the non-negative quadrant. This, together with several other quantile curves, is displayed in Fig. 1. 


$$
\begin{aligned}
\psi\left(s_{1}, s_{2}\right)= & \frac{\left[\left(s_{1}+3\right)\left(2 s_{1}+1\right)+\sqrt{\left(3 s_{1}+9\right)\left(1+3 s_{1}\right)}\right]\left[\left(s_{1}+3\right)\left(2 s_{1}+1\right)+\sqrt{\left(s_{1}+3\right)\left(3 s_{1}+13\right)}\right]}{\left[\left(s_{1}+3\right)\left(2 s_{1}+2 s_{2}+1\right)+\sqrt{\left(3 s_{1}+9\right)\left(1+3 s_{1}\right)}\right]\left[\left(s_{1}+3\right)\left(2 s_{1}+2 s_{2}+1\right)+\sqrt{\left(s_{1}+3\right)\left(3 s_{1}+13\right)}\right]} \\
& \cdot \frac{\left|v_{1}\right|^{2}}{36} \frac{\left(s_{1}+s_{2}+2\right)^{2}\left(s_{1}+3\right)^{2}}{\left(s_{1}^{2}+4 s_{1}+1\right)\left(s_{1}-v_{1}\right)\left(s_{1}-\bar{v}_{1}\right)}
\end{aligned}
$$

Box I.

Table 1

\begin{tabular}{|c|c|c|c|c|c|c|c|c|c|c|}
\hline$\left(x_{1}, x_{2}\right)$ & $(0,0)$ & $(2,0)$ & $(2,2)$ & $(4,0)$ & $(4,2)$ & $(4,4)$ & $(6,0)$ & $(6,2)$ & $(6,4)$ & $(6,6)$ \\
\hline$\hat{R}\left(x_{1}, x_{2}\right)$ & 0.423 & 0.297 & 0.060 & 0.180 & 0.050 & 0.008 & 0.107 & 0.034 & 0.007 & 0.001 \\
\hline$R\left(x_{1}, x_{2}\right)$ & 0.424 & 0.301 & 0.060 & 0.184 & 0.050 & 0.008 & 0.110 & 0.035 & 0.007 & 0.001 \\
\hline
\end{tabular}

Comparison between the simulated ruin function $(\hat{R})$ and the inverted function $(R)$ for various values of the initial capital $\left(x_{1}, x_{2}\right)$.

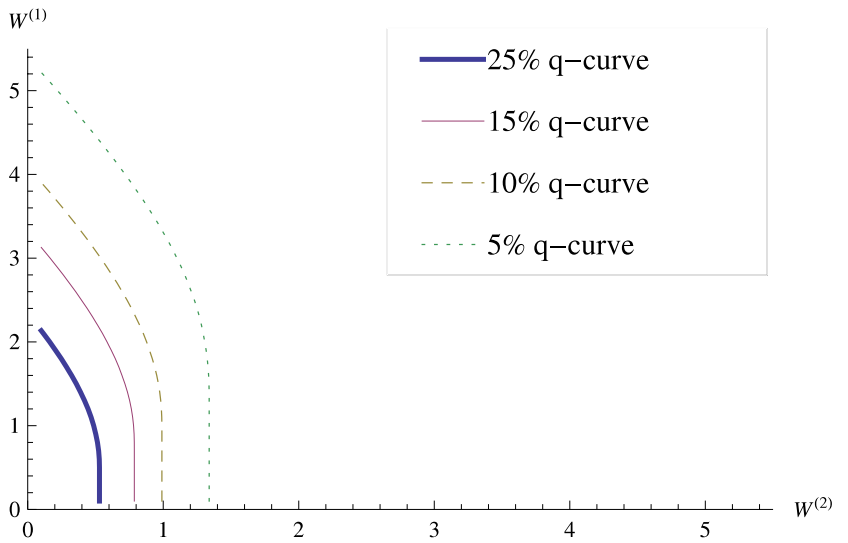

Fig. 1. $25 \%, 15 \%, 10 \%$, and respectively $5 \%$-quantile curves for the ruin function in Example 1. The abscissa corresponds to the values at risk in the second insurance line/the marginal tail of $W^{(2)}$.

Finally, as a verification, we estimated the ruin function using simulation. Upon choosing suitable bin sizes that account for the atom at $(0,0)$ of $\left(W^{(1)}, W^{(2)}\right)$, the uniform distance between the output of the inversion algorithm and the simulated ruin function is of the order of $10^{-3}$. If we denote with $R\left(x_{1}, x_{2}\right)=$ $\mathbb{P}\left(\tau_{\vee}\left(x_{1}, x_{2}\right)<\infty\right)$, the joint ruin function, the simulation comparison is in Table 1.

Remark 10. The quantile curve plots in Fig. 1 contain lines which below the main diagonal are straight. This is a consequence of the ordering assumption on the claims (and implicitly on the waiting times) since we can write for $x_{1} \leq x_{2}$

$\mathbb{P}\left(W^{(1)}>x_{1}, W^{(2)}>x_{2}\right)=\mathbb{P}\left(W^{(2)}>x_{2}\right)$,

because $W^{(2)}>x_{2}$ implies $W^{(1)}>x_{1}$ for all $x_{1} \leq x_{2}$.

Example 2. The parameters are the same as in Example 1, except now the order is $n=3$. The kernel is

$$
\begin{aligned}
& \tilde{K}\left(s_{1}, z\right)=1-\frac{72}{\left(3+s_{1}\right)^{3}(1-z)^{3}(2+z)^{3}} \\
& -\frac{12}{\left(3+s_{1}\right)^{2}(1-z)^{2}(2+z)^{2}}-\frac{2}{\left(3+s_{1}\right)(1-z)(2+z)} .
\end{aligned}
$$

In Box II we list the zeros of the numerator. The radicals are again defined when the cut is taken along the negative half of the real axis.

Remark 11. The above formulae cannot be simplified. When choosing a branch for the square root as a function of a complex variable, one has for $a \neq b$ :

$\sqrt{(z-a)(z-b)} \neq \sqrt{z-a} \sqrt{z-b}$.
In addition, the term $\sqrt{-\left(3+s_{1}\right)^{2}}$ is discontinuous (its discontinuity line is $\ell m s_{1}=0$ ) and it contributes towards the discontinuities of the zeros $v_{i}\left(s_{1}\right), i=\overline{1,4}$.

It is not clear a priori which one of the four zeros to choose when constructing the one-parameter factor $\tilde{K}_{s_{1}}(z)$ from (5.7) because, in contrast to Example 1, the branch cuts of $v_{1}\left(s_{1}\right)$ up to $v_{4}\left(s_{1}\right)$ cross inside the right half-plane, and the zeros $v_{i}\left(s_{1}\right)$ jump from positive to negative real values when the argument passes between the regions bounded by the cuts in $\mathcal{R} e s_{1}>0$.

The key observation is that $v_{1}\left(s_{1}\right)$ is an analytic continuation of $v_{2}\left(s_{1}\right)$, and $v_{3}\left(s_{1}\right)$ is an analytic continuation for $v_{4}\left(s_{1}\right)$. Therefore, in order to obtain $\tilde{K}_{s_{1}}(z)$, one has to glue together (using analytic continuation) the negative branches of $v_{1}\left(s_{1}\right)$ and $v_{2}\left(s_{1}\right)$ on the one hand, and of $v_{3}\left(s_{1}\right)$ and $v_{4}\left(s_{1}\right)$ on the other, for $\mathcal{R e} s_{1}>0 . v_{5}\left(s_{1}\right)$ is negative for any $\operatorname{Re}_{1}>0$ so it will always enter the formula for $K_{s_{1}}^{+}(\cdot)$ as opposed to $v_{6}\left(s_{1}\right)$ which is positive and does not play any role. Moreover the branch cuts of $v_{1}\left(s_{1}\right)$ up to $v_{4}\left(s_{1}\right)$ partition the complex half-plane in 4 regions symmetric around the real axis and the cuts are pairwise parallel lines at angles $\pm \pi / 4$. To be more precise, we have to use the 3 different branches of $\tilde{K}_{s_{1}}^{+}(z)$ :

$\tilde{K}_{s_{1}}^{1,3}(z)=\frac{\left(z-v_{1}\left(s_{1}\right)\right)\left(z-v_{3}\left(s_{1}\right)\right)\left(z-v_{5}\left(s_{1}\right)\right)}{(z+1)^{3}}$,
$\tilde{K}_{s_{1}}^{2,3}(z)=\frac{\left(z-v_{2}\left(s_{1}\right)\right)\left(z-v_{3}\left(s_{1}\right)\right)\left(z-v_{5}\left(s_{1}\right)\right)}{(z+1)^{3}}$,
$\tilde{K}_{s_{1}}^{2,4}(v)=\frac{\left(z-v_{2}\left(s_{1}\right)\right)\left(z-v_{4}\left(s_{1}\right)\right)\left(z-v_{5}\left(s_{1}\right)\right)}{(z+1)^{3}}$.

The branches $C^{1,3}\left(s_{1}\right), C^{2,3}\left(s_{1}\right)$ and $C^{2,4}\left(s_{1}\right)$ are obtained similarly because from (5.13), these are related to the corresponding branches of $\tilde{K}_{s_{1}}^{+}(z)$ by setting $z=s_{1}$; the branch cuts and the partition of the complex plane are therefore the same. Since both $C(\cdot)$ and $K_{s_{1}}^{+}(\cdot)$ enter Formula (5.9), the expression for the LST of the survival function/joint waiting time (see Remark 3 ) is obtained by patching together (via analytic continuation) the positive branches (in the $s_{1}$-plane) of the generalized Wiener-Hopf factors from Proposition 2 and Theorem 2.

In Fig. 2 we plot a section in the three branches of the real part of the LST of the survival measure in Example 2. More precisely consider the section

$\zeta(y):=\operatorname{Re} \psi(i y, 14+i y)$

that runs along the imaginary axis in the first argument $s_{1}$ (the argument that generates the discontinuities). From this figure it becomes apparent how the three different branches of $\zeta(y)$ are continuations of each other: the central branch belongs to $\operatorname{Re} \psi^{1,3}(i y, 14+i y)$ (the blue curve). This is continued by the branch $\mathcal{R e} \psi^{2,3}(i y, 14+i y$ ) (the dashed red curve) which in turn 


$$
\begin{aligned}
& v_{1}\left(s_{1}\right)=\frac{-\left(s_{1}+3\right)^{2}-\sqrt{\left(s_{1}+3\right)^{4}-4\left(s_{1}+3\right)^{2}\left(-24-14 s_{1}-2 s_{1}^{2}+2 \sqrt{2} \sqrt{-\left(3+s_{1}\right)^{2}}\right)}}{2\left(s_{1}+3\right)^{2}}, \\
& v_{2}\left(s_{1}\right)=\frac{-\left(s_{1}+3\right)^{2}+\sqrt{\left(s_{1}+3\right)^{4}-4\left(s_{1}+3\right)^{2}\left(-24-14 s_{1}-2 s_{1}^{2}+2 \sqrt{2} \sqrt{-\left(3+s_{1}\right)^{2}}\right)}}{2\left(s_{1}+3\right)^{2}}, \\
& v_{3}\left(s_{1}\right)=\frac{-\left(s_{1}+3\right)^{2}-\sqrt{\left(s_{1}+3\right)^{4}-4\left(s_{1}+3\right)^{2}\left(-24-14 s_{1}-2 s_{1}^{2}-2 \sqrt{2} \sqrt{-\left(3+s_{1}\right)^{2}}\right)}}{2\left(s_{1}+3\right)^{2}}, \\
& v_{4}\left(s_{1}\right)=\frac{-\left(s_{1}+3\right)^{2}+\sqrt{\left(s_{1}+3\right)^{4}-4\left(s_{1}+3\right)^{2}\left(-24-14 s_{1}-2 s_{1}^{2}-2 \sqrt{2} \sqrt{-\left(3+s_{1}\right)^{2}}\right)}}{2\left(s_{1}+3\right)^{2}}, \\
& v_{5}\left(s_{1}\right)=\frac{-\left(s_{1}+3\right)-\sqrt{3} \sqrt{\left(s_{1}+3\right)\left(1+3 s_{1}\right)}}{2\left(s_{1}+3\right)} \\
& v_{6}\left(s_{1}\right)=\frac{-\left(s_{1}+3\right)+\sqrt{3} \sqrt{\left(s_{1}+3\right)\left(1+3 s_{1}\right)}}{2\left(s_{1}+3\right)}
\end{aligned}
$$

Box II.

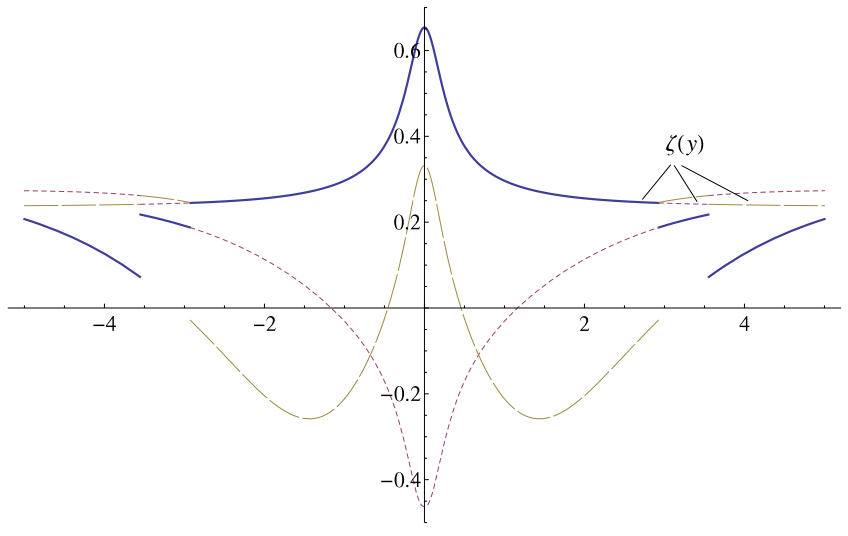

Fig. 2. The plot of the three branches of the section $\zeta(y)$ from (6.5).

is continued by $\operatorname{Re} \psi^{2,4}(i y, 14+i y)$ (the orange curve segment) towards the ends of the plot.

We numerically inverted the expression obtained for the transform $\psi\left(s_{1}, s_{2}\right)$ using den Iseger's algorithm (den Iseger, 2006). Again, the division size is $\Delta=.1$ and the grid size is $M=2^{6}$ in both directions. In Fig. 3(a) the plot of the ruin function $\mathbb{P}\left(\tau_{\vee}(\cdot, \cdot)<\infty\right)$ is presented (see Formula (2.2) and Remark 2) or equivalently the tail of the equilibrium distribution for the bivariate waiting time

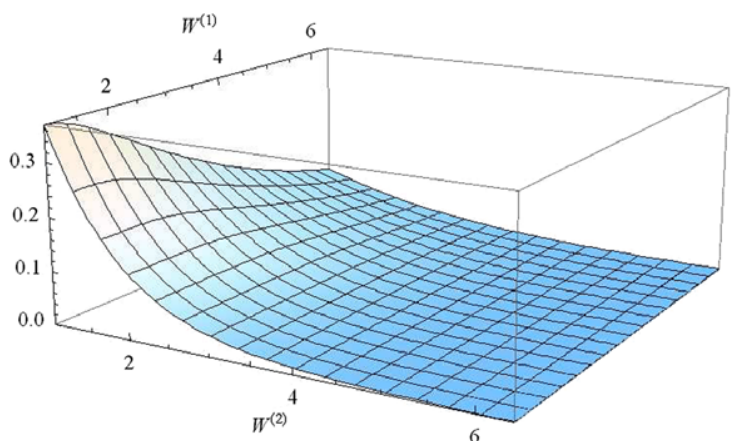

(a) The joint ruin function/the bivariate tail of the waiting time from Example 2.
$\left(W^{(1)}, W^{(2)}\right)($ Remark 3$)$ in Example 2. We can write in general

$$
\begin{aligned}
\mathbb{P}\left(W^{(1)}>0, W^{(2)}>0\right)= & 1-\mathbb{P}\left(W^{(1)}=0\right)-\mathbb{P}\left(W^{(2)}=0\right) \\
& +\mathbb{P}\left(W^{(1)}=0, W^{(2)}=0\right),
\end{aligned}
$$

and because of the ordering, $\mathbb{P}\left(W^{(1)}=0, W^{(2)}=0\right)=$ $\mathbb{P}\left(W^{(1)}=0\right)$. And then the value at $(0,0)$ of the joint ruin function is $\mathbb{P}\left(W^{(2)}>0\right)$, which in this example approximately equates 0.37 .

Finally, in Fig. 3(b) we present various quantile curves for the ruin function/stationary tail of the waiting time.

Comparison of risks. In Fig. 4 we compare the results for quantile curves of the ruin function of Example 1 with the quantile curves of the ruin function for the case where the input is decoupled. By this we mean we take three samples $N_{1}, N_{2}$ and $N_{3}$ from a uniform distribution on $\{1,2\}$ and define the random variables $A_{\text {dec }}=\sum_{i=1}^{N_{1}} A_{i}, D_{\text {dec }}=\sum_{i=1}^{N_{2}} D_{i}, B_{\text {dec }}^{(2)}=\sum_{i=1}^{N_{3}} B_{i}^{(2)}$, where $\left(A_{i}\right)_{i \leq n},\left(B_{i}^{(2)}\right)_{i \leq n}$, and $\left(D_{i}\right)_{i \leq n}$ are mutually independent sequences of exponential random variables with rates $\lambda, \mu$ and $\mu_{D}$ respectively. In this case the inter-arrival time becomes independent of the claim size vector, while marginally $A_{d e c}, B_{d e c}^{(2)}$ and $B_{d e c}^{(1)}$ have the same distribution as in Example 1. The kernel for this instance is

$$
\begin{aligned}
\tilde{K}\left(s_{1}, z\right)= & \left(\frac{9}{2\left(3+s_{1}\right)^{2}}+\frac{3}{2\left(3+s_{1}\right)}\right) \\
& \times\left(\frac{1}{2(1-z)^{2}}+\frac{1}{2(1-z)}\right)\left(\frac{2}{(2+z)^{2}}+\frac{1}{2+z}\right) .
\end{aligned}
$$

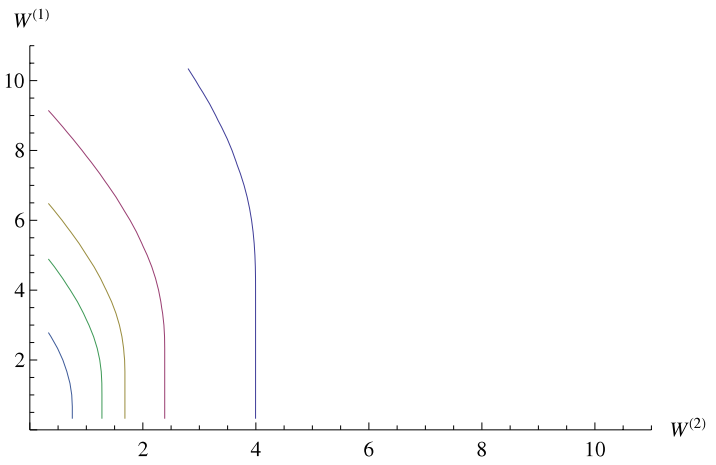

(b) $25 \%, 15 \%, 10 \%, 5 \%$, respectively $1 \%$-quantile curves for the ruin function in Example 2. The $x$-axis corresponds to the marginal tail of $W^{(2)}$.

Fig. 3. Numerical results for the reserve process in Example 2: (a) ruin function and (b) quantile curves. 


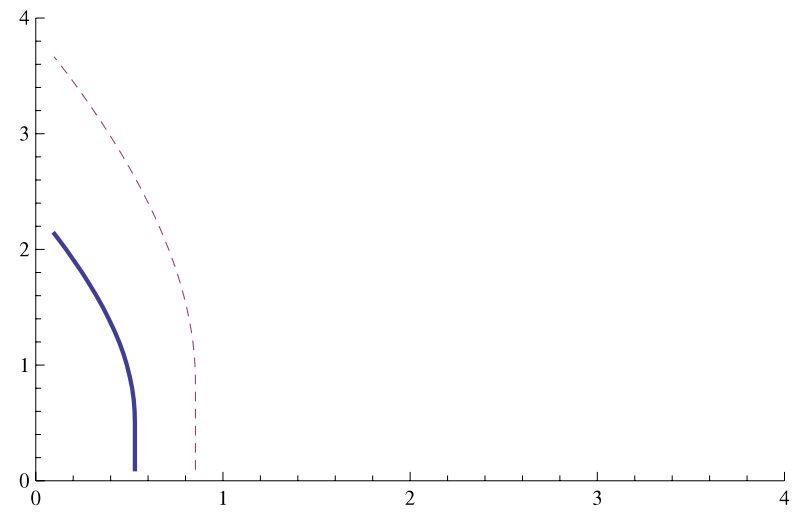

(a) $25 \%$ quantile curves.

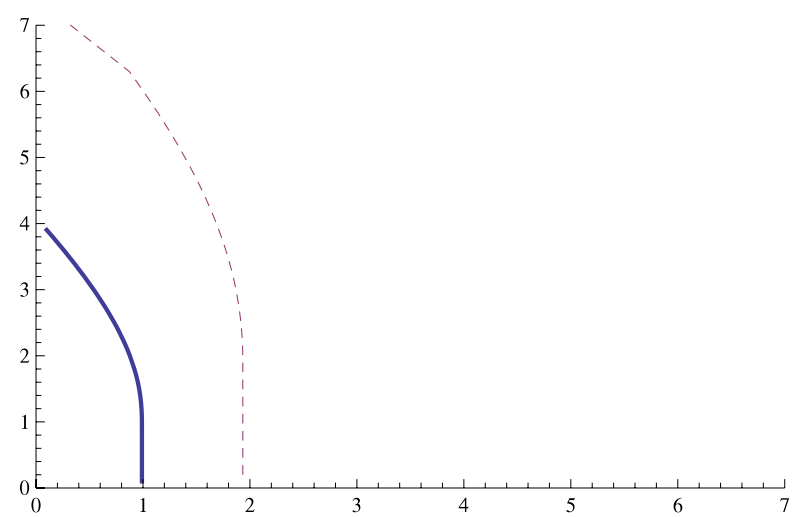

(c) $10 \%$ quantile curves.

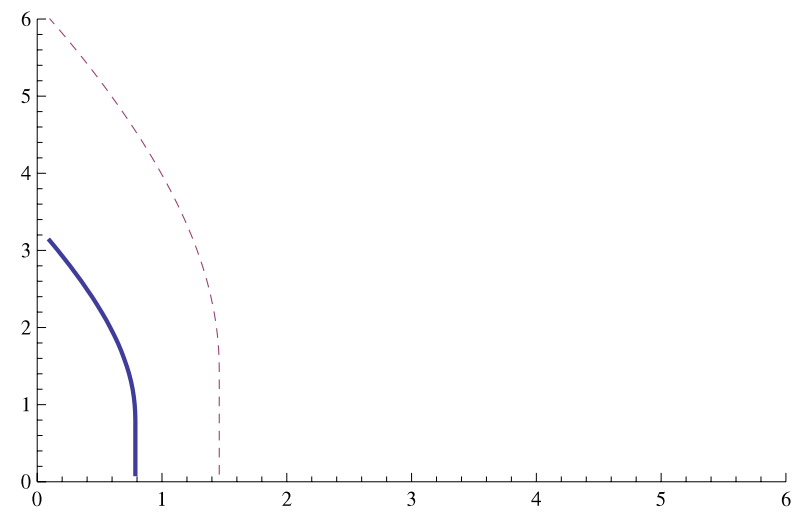

(b) $15 \%$ quantile curves.

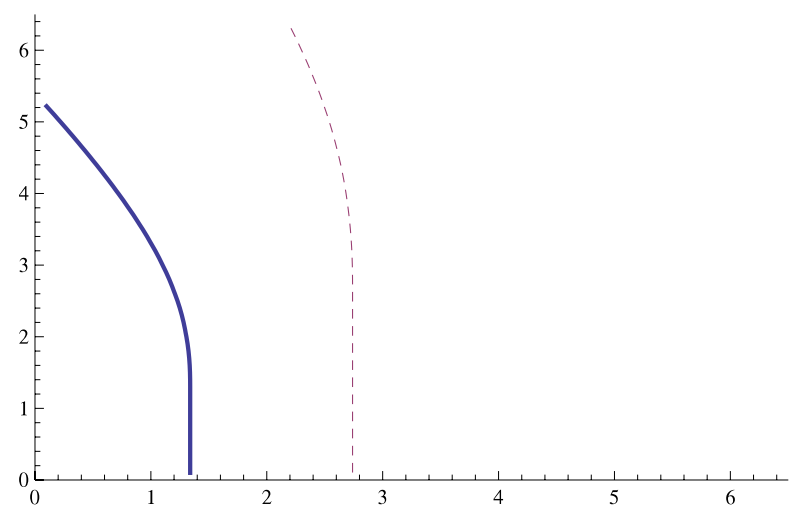

(d) $5 \%$ quantile curves.

Fig. 4. Comparison of risks. Dashed curves correspond to decoupled input.

The zeros of the numerator as a polynomial in $z$ are already too complicated to present here. This instance is similar to Example 2 in terms of the analytic behaviour of these zeros.

The main point is that, similarly as in Badila et al. (2013), numerical data suggests that the ruin functions corresponding to positively correlated input on the one hand, and the ruin functions for decoupled input on the other are stochastically ordered (Fig. 4).

Example 3 (Proportional Reinsurance). This is the case with proportional claims. There is a common arrival process such that the inter-arrival time $A_{n}$ is correlated with the claim size $B_{n}$, and $\alpha B_{n}$ is deducted from the first insurance line and $(1-\alpha) B_{n}$ from the second.

We take $N \sim \operatorname{Unif}\{1,2,3\}, \lambda=\mu=1, \alpha=3 / 4$ and unit income rates. For the purpose of comparing risks, we will consider three instances for the random vector $(A, B)$ :

positive correlation: $(A, B)_{p o s} \sim(\operatorname{Erlang}(N, \lambda), \operatorname{Erlang}(N, \mu))$, independence: $(A, B)_{0} \sim\left(\operatorname{Erlang}\left(N_{1}, \lambda\right)\right.$, Erlang $\left.\left(N_{2}, \mu\right)\right)$, with $N_{1}, N_{2}$ two copies of $N$.

negative correlation: $(A, B)_{n e g} \sim(\operatorname{Erlang}(N, \lambda), \operatorname{Erlang}(4-$ $N, \mu)$ ).

The kernels corresponding to these instances are

$$
\begin{aligned}
\tilde{K}_{p o s}\left(s_{1}, z\right)= & \frac{1}{3(1-z)\left(1+s_{1} / 2+z / 4\right)} \\
& +\frac{1}{3(1-z)^{2}\left(1+s_{1} / 2+z / 4\right)^{2}} \\
& +\frac{1}{3(1-z)^{3}\left(1+s_{1} / 2+z / 4\right)^{3}},
\end{aligned}
$$

$$
\begin{aligned}
\tilde{K}_{0}\left(s_{1}, z\right)= & \left(\frac{1}{3(1-z)}+\frac{1}{3(1-z)^{2}}+\frac{1}{3(1-z)^{3}}\right) \\
& \times\left(\frac{1}{3\left(1+s_{1} / 2+z / 4\right)}+\frac{1}{3\left(1+s_{1} / 2+z / 4\right)^{2}}\right. \\
& \left.+\frac{1}{3\left(1+s_{1} / 2+z / 4\right)^{3}}\right) \\
\tilde{K}_{n e g}\left(s_{1}, z\right)= & \frac{1}{3(1-z)\left(1+s_{1} / 2+z / 4\right)^{3}} \\
& +\frac{1}{3(1-z)^{2}\left(1+s_{1} / 2+z / 4\right)^{2}} \\
& +\frac{1}{3(1-z)^{3}\left(1+s_{1} / 2+z / 4\right)} .
\end{aligned}
$$

These functions stand for $\mathbb{E} e^{-\left[s_{1}(2 \alpha-1)+z(1-\alpha)\right] B+z A}$ under the three couplings. The correlations between the variables $A$ and $B$ can also be read directly from the shapes of these transforms.

Numerical illustrations are in Fig. 5.

Comparison of risks In the table below we present various points at which a specific ruin probability is achieved. Given a fixed value at risk, any value for starting capital $\left(x_{1}, x_{2}\right)$ lying on the respective quantile curve will achieve this. Interestingly, the risks are ordered between the various types of correlations (see also Fig. 6). Let us denote by $R\left(x_{1}, x_{2}\right)=\mathbb{P}\left(\tau_{\vee}\left(x_{1}, x_{2}\right)<\infty\right)$, the probability that eventually, both lines are ruined. Then for the three types of correlation we give the values of the function $R$ in Table 2. Positive correlation gives the lowest ruin probabilities for any starting capital considered. 

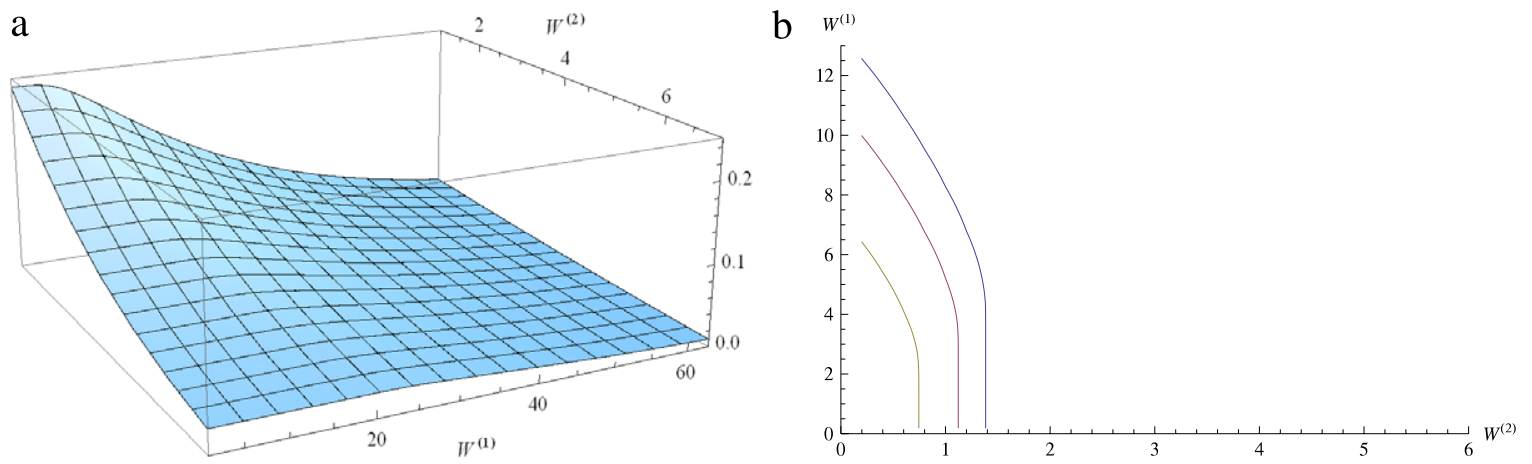

Fig. 5. Bivariate tail (left) and respectively $10 \%, 5 \%$, and $3 \%$ quantile curves (right) for proportional reinsurance with negative correlation.

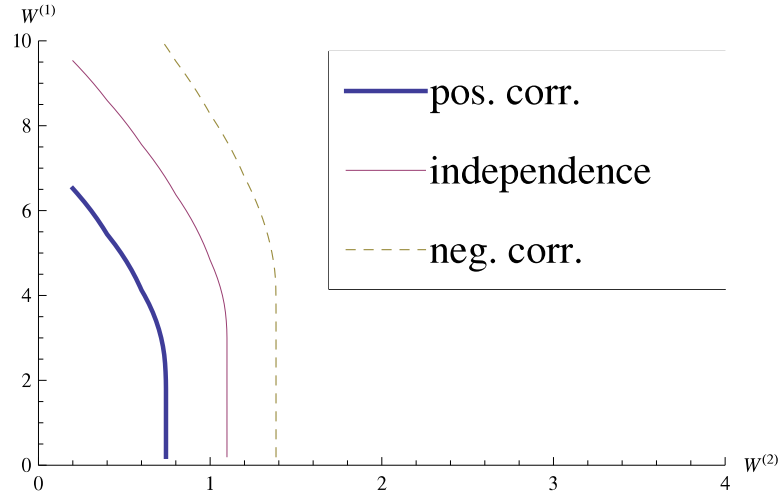

(a) $3 \%$ quantile curves.

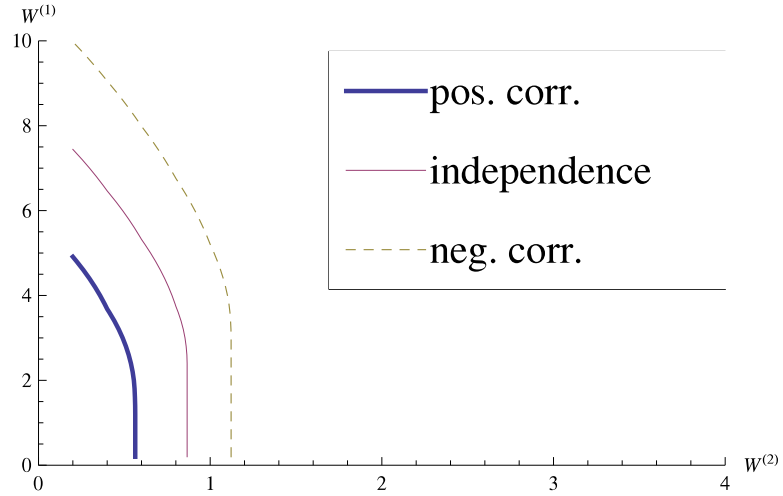

(b) $5 \%$ quantile curves.

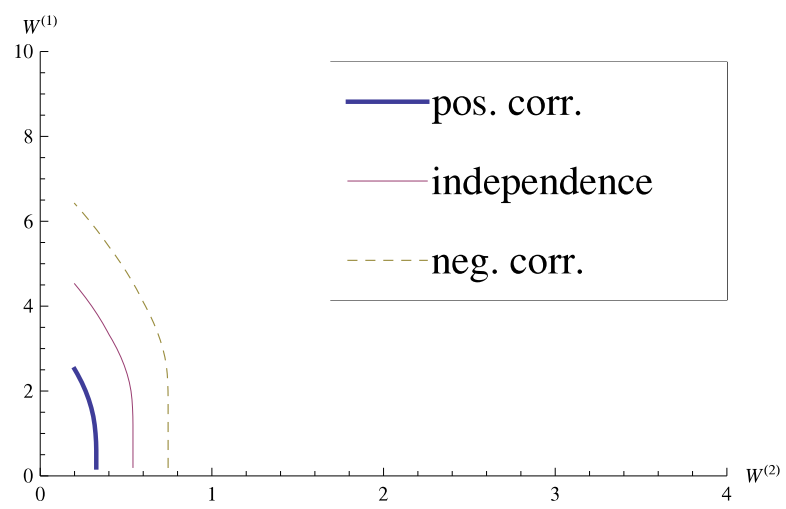

(c) $10 \%$ quantile curves.

Fig. 6. Comparison of risks.

Table 2

Comparison between the joint ruin functions $R_{n e g}, R_{0}$ and $R_{\text {pos }}$ respectively, for the various types of correlation.

\begin{tabular}{|c|c|c|c|c|c|c|c|c|c|}
\hline$\left(x_{1}, x_{2}\right)$ & $(0,0)$ & $(2.4,0)$ & $(4.8,0)$ & $(4.8,0.4)$ & $(6.4,0.4)$ & $(6.4,0.8)$ & $(9,0.4)$ & $(9,0.8)$ & $(11.8,0.8)$ \\
\hline$R_{\text {neg }}\left(x_{1}, x_{2}\right)$ & 0.2388 & 0.1995 & 0.1309 & 0.0862 & 0.0648 & 0.0402 & 0.0397 & 0.0253 & 0.0149 \\
\hline$R_{0}\left(x_{1}, x_{2}\right)$ & 0.1922 & 0.1516 & 0.0896 & 0.0536 & 0.0375 & 0.0214 & 0.0203 & 0.0120 & 0.0061 \\
\hline$R_{p o s}\left(x_{1}, x_{2}\right)$ & 0.1381 & 0.0979 & 0.0486 & 0.0237 & 0.0148 & 0.0070 & 0.0065 & 0.0033 & 0.0013 \\
\hline
\end{tabular}

\section{Conclusions}

The working assumptions of rationality and ordering for the trivariate input made up from the generic claim vector together with the preceding inter-arrival time, allow one to obtain detailed numerical results, for the joint ruin probability as a function of the initial reserves. Our numerical results suggest that when comparing ruin functions that correspond to various correlation structures between claim intervals and claim sizes, positive correlation among these cause lower values of the ruin probability compared to zero correlation, and even more so, when compared to negative correlation.

By using the relations described in Section 2, one can recover the other types of ruin/survival functions, where the numerical inversion of the marginal transforms, when needed, can be carried out using the one-dimensional inversion algorithm.

\section{Acknowledgements}

The first author was supported by Project 613.001.017 of the Netherlands Organisation for Scientific Research (NWO). The 
second and third authors were supported by the IAP BESTCOM Project funded by the Belgian government.

\section{Appendix}

Proposition A.1 (On the zeros of the Kernel $1-\tilde{K}\left(s_{1}, z\right)$ ). For each $s_{1}$, Re $s_{1} \geq 0, g\left(s_{1}, z\right)-f\left(s_{1}, z\right)$ and $g\left(s_{1}, z\right)$ have the same number of zeros in $\mathcal{R} e z \geq 0$.

Proof. We show that $g\left(s_{1}, z\right)$ dominates $f\left(s_{1}, z\right)$ on a suitably chosen contour in the complex $z$-plane. From this the claim in the proposition will follow via Rouché's theorem (Titchmarsh, 1939, p. 116). Consider the contour which is made up from the extended arc:

$\mathcal{C}_{\epsilon}:=\left\{r e^{i \varphi} ; \varphi \in[-\pi / 2-\arccos \epsilon, \pi / 2+\arccos \epsilon]\right\}$,

together with the line segment

$I:=\left\{-\epsilon+i \omega ;|\omega| \in\left[0, r \sqrt{1-\epsilon^{2}}\right]\right\}$.

The rationality of the transform ensures that $\tilde{K}\left(s_{1}, z\right)$ can be analytically continued on a thin strip: $\mathcal{R e} z<0$, $|\mathcal{R} e z|<\epsilon$. We first consider the contour $\mathcal{C}_{\epsilon}$. On the one hand we have the triangle inequality for $f:\left|f\left(s_{1}, z\right)\right| \leq \bar{f}\left(\left|s_{1}\right|,|z|\right)$, where $\bar{f}$ is a polynomial with the same degree as $f$. On the other hand, if we represent $g_{s_{1}}(z)$ for fixed $s_{1}$ as $g_{s_{1}}(z)=a_{m}\left(s_{1}\right) \prod_{i}\left(z-\xi_{i}\left(s_{1}\right)\right)$, with $\xi_{i}\left(s_{1}\right)$ its zeros and $a_{m}\left(s_{1}\right)$ the coefficient of $z^{m}, m=m\left(s_{1}\right)=\operatorname{deg} g_{s_{1}}(z)$, then the same triangle inequality gives a lower bound for $\left|g\left(s_{1}, z\right)\right|$ :

$$
\begin{aligned}
\left|g\left(s_{1}, z\right)\right| & =\left|a_{m}\left(s_{1}\right)\right| \prod_{i}\left|z-\xi_{i}\left(s_{1}\right)\right| \\
& \geq\left|a_{m}\left(s_{1}\right)\right| \prod_{i}\left(|z|-\left|\xi_{i}\left(s_{1}\right)\right|\right)=: \bar{g}\left(s_{1},|z|\right),
\end{aligned}
$$

with the remark that $\bar{g}_{s_{1}}(|z|)$ has the same degree as $g_{s_{1}}(z)$.

Now we can bound for $r$ sufficiently large, such that the interior of $\mathcal{C}_{\epsilon} \cup I$ contains all the zeros of $\bar{g}_{s_{1}}(|z|)$ and $z \in \mathcal{C}_{\epsilon}$ :

$$
\left|\frac{f\left(s_{1}, z\right)}{g\left(s_{1}, z\right)}\right| \leq \frac{\bar{f}\left(\left|s_{1}\right|,|z|\right)}{\bar{g}\left(s_{1},|z|\right)} \rightarrow 0, \quad \text { as } r \rightarrow \infty .
$$

Convergence holds because the degree of the numerator is strictly less than that of the denominator, by Assumption 2.1. This establishes the bound $\left|g\left(s_{1}, z\right)\right|>\left|f\left(s_{1}, z\right)\right|$ on $\mathcal{C}_{\epsilon}$, for $r$ large enough.

For the segment $I$, we use the safety loading condition for the second(!) line: $c^{(2)} \mathbb{E} A-\mathbb{E} B^{(2)}>0$. That is, we start with the fact $\left.\frac{\mathrm{d}}{\mathrm{d} z} \frac{f(0, z)}{g(0, z)}\right|_{z=0}=c^{(2)} \mathbb{E} A-\mathbb{E} B^{(2)}>0$. So for $\epsilon>0$ sufficiently small, $\frac{f(0,-\epsilon)}{g(0,-\epsilon)}<\frac{f(0,0)}{g(0,0)}=1$. Then we can write for $z \in I$ :

$$
\begin{aligned}
\left|\frac{f\left(s_{1}, \epsilon+i \omega\right)}{g\left(s_{1}, \epsilon+i \omega\right)}\right| & \leq \mathbb{E}\left(\left|e^{-s_{1} D}\right| \cdot\left|e^{-\epsilon\left(A-B^{(2)}\right)}\right| \cdot\left|e^{-i \omega\left(A-B^{(2)}\right)}\right|\right) \\
& \leq \mathbb{E} e^{-\epsilon\left(A-B^{(2)}\right)}=\frac{f(0,-\epsilon)}{g(0,-\epsilon)}<1 .
\end{aligned}
$$

Above we used the rough bound $\left|e^{-s_{1} D}\right| \leq 1$. This completes the proof.

Notice that the key role in the proof is played by $\rho_{2}<1$ and not $\rho_{1}<1\left(\rho_{2}<\rho_{1}\right)$

\section{References}

Asmussen, S., Albrecher, H., 2010. Ruin Probabilities. World Scientific Publ. Cy, Singapore.

Avram, F., Palmowski, Z., Pistorius, M., 2008a. A two-dimensional ruin problem on the positive quadrant. Insurance Math. Econom. 42 (1), 227-234.

Avram, F., Palmowski, Z., Pistorius, M., 2008b. Exit problem of a two-dimensional risk process from the quadrant: exact and asymptotic results. Ann. Appl. Probab. 18, 2421-2449.

Badescu, A.L., Cheung, E.C.K., Rabehasaina, L., 2011. A two dimensional risk model with proportional reinsurance. J. Appl. Probab. 48, 749-765.

Badila, E.S., Boxma, O.J., Resing, J.A.C., 2013. Queues and risk processes with dependencies. Stoch. Models 30 (3), 390-419.

Badila, E.S., Boxma, O.J., Resing, J.A.C., 2014. Queues and risk models with simultaneous arrivals. Adv. Appl. Probab. 46 (3), 812-831.

Bladt, M., Nielsen, B.F., 2010. Multivariate matrix-exponential distributions. Stoch. Models 26 (1), 1-26.

Cai, J., Li, H., 2005. Multivariate risk model of phase type. Insurance Math. Econom. 36, 137-152.

Chan, W.-S., Yang, H., Zhang, L., 2003. Some results on ruin probabilities in a twodimensional risk model. Insurance Math. Econom. 32, 345-358.

Cohen, J.W., 1982. The Single Server Queue. North Holland.

Cohen, J.W., Boxma, O.J., 1983. Boundary Value Problems in Queueing System Analysis. North-Holland Publ. Cy, Amsterdam.

den Iseger, P., 2006. Numerical transform inversion using Gaussian quadrature. Probab. Engrg. Inform. Sci. 20, 1-44.

Gong, L., Badescu, A.L., Cheung, E.C.K., 2012. Recursive methods for a multidimensional risk process with common shocks. Insurance Math. Econom. 50, $109-120$.

Kingman, J.F.C., 1966. On the algebra of queues. J. Appl. Probab. 3 (2), 285-386.

Kulkarni, V.G., 1989. A new class of multivariate phase type distributions. Oper. Res. 37 (1), 151-158.

Prabhu, N.U., 1980. Stochastic Storage Processes. Queues, Insurance Risk, and Dams. Springer Verlag.

Sundt, B., 1999. On multivariate Panjer recursions. Astin Bull. 29, 29-45.

Titchmarsh, E.C., 1939. The Theory of Functions, second ed. Oxford University Press. 